\title{
Psychometric properties of self-reported measures of health-related quality of life in people living with HIV: a systematic review
}

Huan Wen ${ }^{1}$, Zhongfang Yang ${ }^{2,3}$, Zheng Zhu ${ }^{2,3^{*}+}\left(\mathbb{0}\right.$, Shuyu Han ${ }^{4}$, Lin Zhang ${ }^{5}$ and Yan $\mathrm{Hu}^{2,3^{*+}}$

\begin{abstract}
Objective: To identify and assess the psychometric properties of patient-reported outcome measures (PROMs) of health-related quality of life (HRQoL) in people living with HIV (PLWH).

Methods: Nine databases were searched from January 1996 to October 2020. Methodological quality was assessed by using the Consensus-based Standards for the Selection of Health Measurement Instruments (COSMIN) Risk of Bias Checklist. We used the COSMIN criteria to summarize and rate the psychometric properties of each PROM. A modified Grading, Recommendations, Assessment, Development, and Evaluation (GRADE) system was used to assess the certainty of evidence.
\end{abstract}

Results: Sixty-nine studies reported on the psychometric properties of 30 identified instruments. All studies were considered to have adequate methodological quality in terms of content validity, construct validity, and internal consistency. Limited information was retrieved on cross-cultural validity, criterion validity, reliability, hypothesis testing, and responsiveness. High-quality evidence on psychometric properties was provided for the Medical Outcomes Study HIV Health Survey (MOS-HIV), the brief version of the World Health Organization's Quality of Life Instrument in HIV Infection (WHOQoL-HIV-BREF), 36-Item Short Form Survey (SF-36), Multidimensional Quality of Life Questionnaire for Persons with HIV/AIDS (MQOL-HIV), and WHOQoL-HIV.

Conclusions: The findings from the included studies highlighted that among HIV-specific and generic HRQoL PROMs, MOS-HIV, WHOQOL-HIV-BREF, SF-36, MQoL-HIV, and WHOQOL-HIV are strongly recommended to evaluate HRQOL in PLWH in research and clinics based on the specific aims of assessments and the response burden for participants.

Keywords: HIV, AIDS, Quality of life, PROM, Systematic review

\section{Introduction}

With the introduction of antiretroviral therapy (ART), the life expectancy of PLWH has been prolonged. However, HIV, ART, infectious diseases, comorbidities, and premature aging pose challenges to the health-related quality of

\footnotetext{
*Correspondence: zhengzhu@fudan.edu.cn; huyan@fudan.edu.cn

${ }^{\dagger}$ Zheng Zhu and Yan Hu contributed equally to this study as

corresponding authors

2 School of Nursing, Fudan University, Shanghai, China

Full list of author information is available at the end of the article
}

life (HRQoL) of PLWH. HRQoL can be defined as one's perceived functioning in the physical, emotional, psychological, and social domains of health [1]. Alternatively, HRQoL was defined by Torrance as a concept incorporating factors that are part of an individual's health [2]. HRQoL is currently regarded as a health aspect of quality of life (QoL); nonhealth aspects, including economic and political circumstances, are not included in HRQoL. Achieving a high level of HRQoL has become an important issue and a component of HIV/AIDS care [3]. In 2016, Lazarus and colleagues proposed adding a original author(s) and the source, provide a link to the Creative Commons licence, and indicate if changes were made. The images or other third party material in this article are included in the article's Creative Commons licence, unless indicated otherwise in a credit line to the material. If material is not included in the article's Creative Commons licence and your intended use is not permitted by statutory regulation or exceeds the permitted use, you will need to obtain permission directly from the copyright holder. To view a copy of this licence, visit http://creativecommons.org/licenses/by/4.0/. The Creative Commons Public Domain Dedication waiver (http://creativeco mmons.org/publicdomain/zero/1.0/) applies to the data made available in this article, unless otherwise stated in a credit line to the data. 
fourth "90" to the existing "90-90-90" target $[4,5]$. The fourth $90 \%$ target is $90 \%$ of PLWH with viral load suppression to have good HRQoL. According to the World Health Organization's 90-90-90-90 goals, improving the HRQoL of PLWH is the ultimate goal of HIV/AIDS treatment and care $[6,7]$. However, which measures are the most suitable is still under debate.

Many HIV-specific and generic HRQoL patientreported outcome measures have been validated in different contexts. As one of the earliest HIV-specific HRQoL PROMs, MOS-HIV is the most commonly used measure [8]. The MOS-HIV consists of 35 items and 10 dimensions, including general health perceptions, physical functioning, role functioning, pain, social functioning, mental health, energy, health distress, cognitive functioning, and overall self-rated quality of life. In addition to MOS-HIV, other HIV-specific HRQoL PROMs are also widely used, including the WHOQoL-HIV-BREF [9], Multidimensional Quality of Life Questionnaire for Persons with HIV/AIDS (MQoL-HIV) [10], HIV Disease Quality of Life 31-Item Instrument (HIV-QL31) [11], and Patient-Reported Outcomes Quality of Life-HIV instrument (PROQoL-HIV) [12]. Additionally, validated subscales or scales with over 40 items, such as the World Health Organization Quality of Life-HIV (WHOQoLHIV) [13], HIV Overview of Problems Evaluation Scale (HOPES) [14], Functional Assessment of HIV Infection (FAHI) [15], HIV/AIDS Targeted Quality of Life (HATQoL) [16], and HIV/AIDS Quality of Life Questionnaire (HIV/AIDSQoL) [17], are also used to evaluate HRQoL. In addition to HIV-specific PROMs, some generic PROMs, including the Short Form Health Survey (SF-12, SF-36) [18, 19], EuroQol-5 Dimensions (EQ-5D) [20, 21], World Health Organization Quality of Life assessment (WHOQoL) [22], Medical Outcomes Study Health Survey (MOS) [23], Missoula-Vitas Quality-of-Life Index (MVQOLI) [24], Patient-Reported Outcomes Measurement Information System (PROMIS) [25], Health Assessment Questionnaire Disability Index (HAQ-DI) [26], Quality of Well-Being scale (QWB) [27], and Health Utility Index 3 (HUI3) [28], have been validated and used in the PLWH population globally. The advantage of using generic HRQoL PROMs is that researchers can directly compare the results with those of other groups based on the same problem without standardizing the data. However, for PLWH, generic PROMs may not be as sensitive as specific PROMs assessing HIV-specific dimensions of HRQoL regarding stigma, relationship issues, and comorbidities [29].

A preliminary literature search was conducted in PubMed, PsycINFO (EBSCO), Cochrane Library (Wiley) and JBI (Ovid), and many reviews on measures of HRQoL were found. Cooper et al. [29] briefly summarized
PROMs with fewer than 40 items for measuring HRQoL in PLWH and found that the MOS-HIV was the most well-established measure. The WHOQoL-HIV-BREF and PROQoL-HIV were considered to have good psychometric properties and to potentially have more relevance to PLWH than other PROMs. However, the study included only instruments that can be completed within $10 \mathrm{~min}$ or that have fewer than 40 items. Additionally, the assessment process of psychometric properties was not systematic enough to provide a concrete conclusion. Clayson et al. [30] conducted reviews with similar aims but in a specific context (in clinical trials and in sub-Saharan Africa) in 2006 and 2010, respectively. Gakhar et al. conducted a nonsystematic review of the literature on quality of life assessment after ART in developed countries in 2013 [31].

However, previous systematic reviews have mainly focused on the content of HRQoL PROMs and have not reported their psychometric properties, which has made it difficult for healthcare professionals to select one of the existing PROMs to evaluate HRQoL in research and clinical practice [29-31]. Accurate and reliable PROMs are a prerequisite for obtaining robust results. It is critical to choose an acceptable PROM with good psychometric properties [32]. Therefore, to obtain reliable evidence regarding the psychometric properties of HRQoL PROMs, we conducted a systematic review to identify and assess the psychometric properties of PROMs of HRQoL in PLWH. This conclusion may provide a scientific basis for researchers to choose PROMs for future scientific research and clinical practice measuring HRQoL in PLWH.

\section{Methods}

\section{Aims and design}

The aim of this study was to identify and assess the psychometric properties of PROMs of HRQoL in PLWH. This systematic review was performed with the guidance of the Joanna Briggs Institute (JBI) methodology for systematic review of psychometric properties and the Preferred Reporting Items for Systematic Reviews and Meta-Analyses (Additional file 1: PRISMA) statement. The protocol of our review was published in JBI Evidence Synthesis [33].

\section{Search strategy}

We conducted a three-step search. First, a limited search was conducted in PubMed to develop search strategies tailored to each database. Second, researchers implemented the search strategies in PubMed, MEDLINE (Ovid), EMBASE (Ovid), CINAHL (EBSCO), Web of Science, ProQuest Dissertations and Theses, Cochrane Library (Wiley), CNKI, and WanFang. The databases 
were searched for published studies from 1st January 1996 to 1st May 2020. We set the start point 1996 because ART was first used in 1996. Google Scholar and Baidu Scholar were searched for gray literature. We used $\mathrm{MeSH}$ terms (["HIV" OR "Acquired Immunodeficiency Syndrome"] AND "Quality of Life") combined with ([HIV OR AIDS OR "acquired immunodeficiency syndrome"] AND "quality of life" AND "COSMIN search filter"). Additional file 2: Appendix I lists the search strategies used for all databases. Finally, we manually reviewed all references included during the supplemental searches.

\section{Inclusion and exclusion criteria}

The inclusion criteria were as follows: (1) studies that targeted HIV-positive adults ( $\geq 18$ years old); (2) studies of any types of self-reported measures, including but not limited to, self-management questionnaires that aimed to measure HRQoL among PLWH; (3) validation studies or studies that aimed to develop PROMs or assess one or more measurement properties; and (4) studies published in either English or Chinese. The exclusion criteria included the following: (1) studies that aimed to validate measures assessing only a certain domain of HRQoL related to specific comorbidities or treatment side effects and (2) studies that provided indirect evidence of psychometric properties (e.g., comparing one PROM with another instrument).

\section{Study screening and selection}

We imported all references identified in the search into Endnote X8 (Clarivate Analytics, PA, USA). After the removal of duplicates, two researchers (HW \& ZY) screened the titles, abstracts, and full texts independently to assess whether the studies met the eligibility criteria. Any discrepancies were resolved by the third researcher (ZZ). The reasons for exclusion of studies at the full-text screening stage were recorded.

\section{Quality appraisal}

Two reviewers (HW \& ZY) assessed the included studies independently by using the COSMIN Risk of Bias Checklist. When there were discrepancies, a third reviewer (ZZ) was included to resolve them. The COSMIN Risk of Bias Checklist consisted of 10 domains (38 items), including PROM development, content validity, structural validity, hypothesis testing of construct validity, cross-cultural validity/measurement invariance, criterion validity, internal consistency, measurement error, test-test reliability, and responsiveness. The options for each item included "very good", "adequate", "doubtful", and "inadequate quality". The methodological quality of the study was based on the worst score counts.

\section{Data extraction and synthesis}

Two researchers (HW \& ZY) independently extracted information, including the author, publication year, country/language, study design, target population, sample size, measurement domains, number of items, and total score range. The main findings regarding psychological properties included construct validity, internal consistency, cross-cultural validity/translation, criterion validity, and reliability. Any discrepancies were discussed between the two researchers.

We used the COSMIN criteria to summarize and rate the psychometric properties of each study regarding structural validity, internal consistency, reliability, measurement error, hypothesis testing for construct validity, cross-cultural validity/measurement invariance, criterion validity, and responsiveness. Each measurement property was rated as sufficient $(+)$, insufficient (-), or indeterminate (?). When data were synthesized and the ratings of each study were consistent, the overall rating of the measurement property was rated as sufficient $(+)$ and insufficient $(-)$. If the ratings of each study were all sufficient $(+)$, the overall rating of the measurement property was rated as sufficient $(+)$. If the ratings of each study were all insufficient $(-)$, the overall rating of the measurement property was rated as insufficient $(-)$. We used narrative synthesis to synthesize the data for each measurement property. If the ratings of each study were inconsistent, we explored possible explanations (e.g., different languages). If the explanation was reasonable, we provided ratings by subgroup. If the explanation was unreasonable, the overall rating of the measurement property was rated as inconsistent $( \pm)$. If there was no information to support the rating, the overall rating was rated as uncertain (?).

\section{Assessment of the certainty of the evidence}

We used a modified Grading of Recommendations, Assessment, Development, and Evaluation (GRADE) system to assess the certainty of the evidence. Each piece of evidence was graded for risk of bias, inconsistency, imprecision, and indirectness. Four reviewers (HW, ZY, ZZ, and SH) graded each measurement property and each PROM separately. Discrepancies were resolved by the fifth reviewer $(\mathrm{YH})$. Based on the methodological quality of each psychometric property, four reviewers finally classified the instruments as strongly 
recommended, weakly recommended and not recommended according to the modified GRADE system. The classification results were verified by all authors.

\section{Results}

\section{Literature search}

The literature screening and selection process is shown in Fig. 1. In the initial search, a total of 13,371 articles were identified in the databases. Twenty-one articles were found through additional supplementary searches. After the removal of duplicates, a total of 10,097 articles were retained, and 10,028 articles were deleted after the review of the titles, abstracts, and full text. We finally included
69 articles [9-28, 34-82]. A total of 30 PROMs were investigated in the included studies.

\section{Study description}

Among the 69 included articles, 54 were in English, and 15 were in Chinese; the articles were published from 1996 to 2019. A description of the studies is shown in Table 1. All the included studies were cross-sectional studies. Twenty studies were conducted in China $[17,22$, $36-40,57-62,74,77-82]$, fourteen in the United States $[15,16,21,25-27,35,42,64,65,67,72,73,75]$, three in Uganda $[24,41,46]$, three in Italy $[44,49,69]$, two in Australia $[70,71]$, two in Vietnam $[20,55]$, two in Portugal

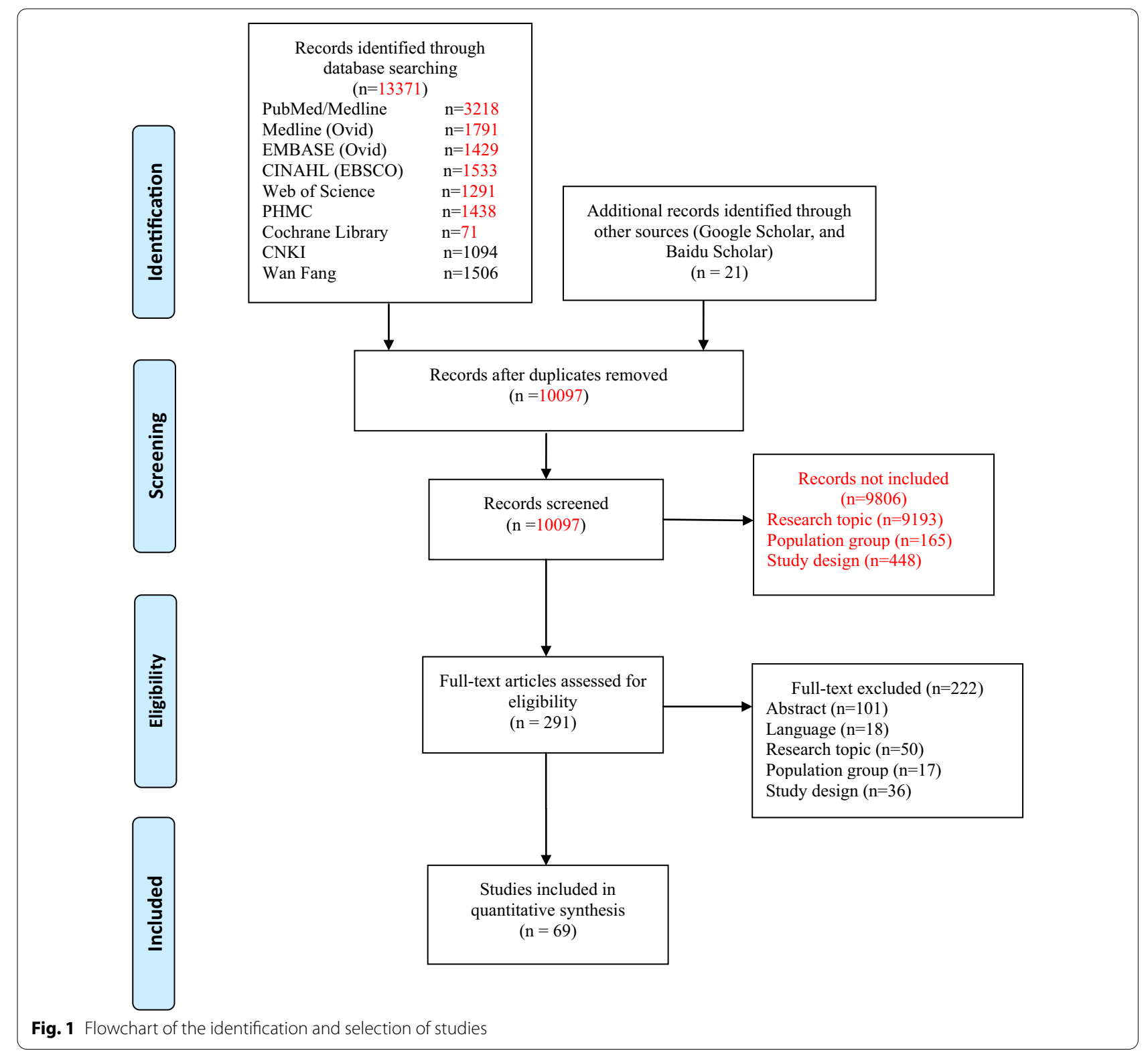




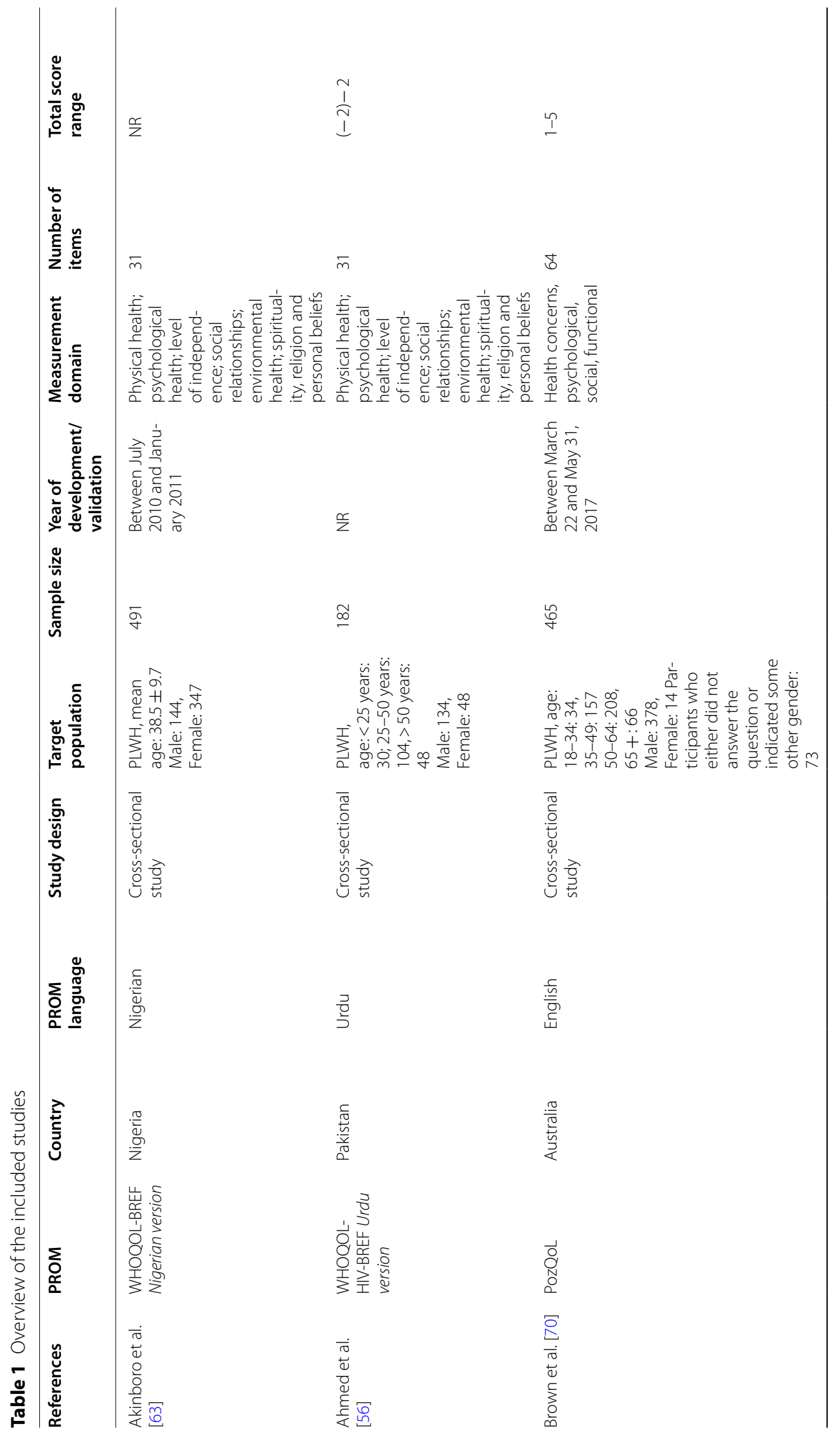




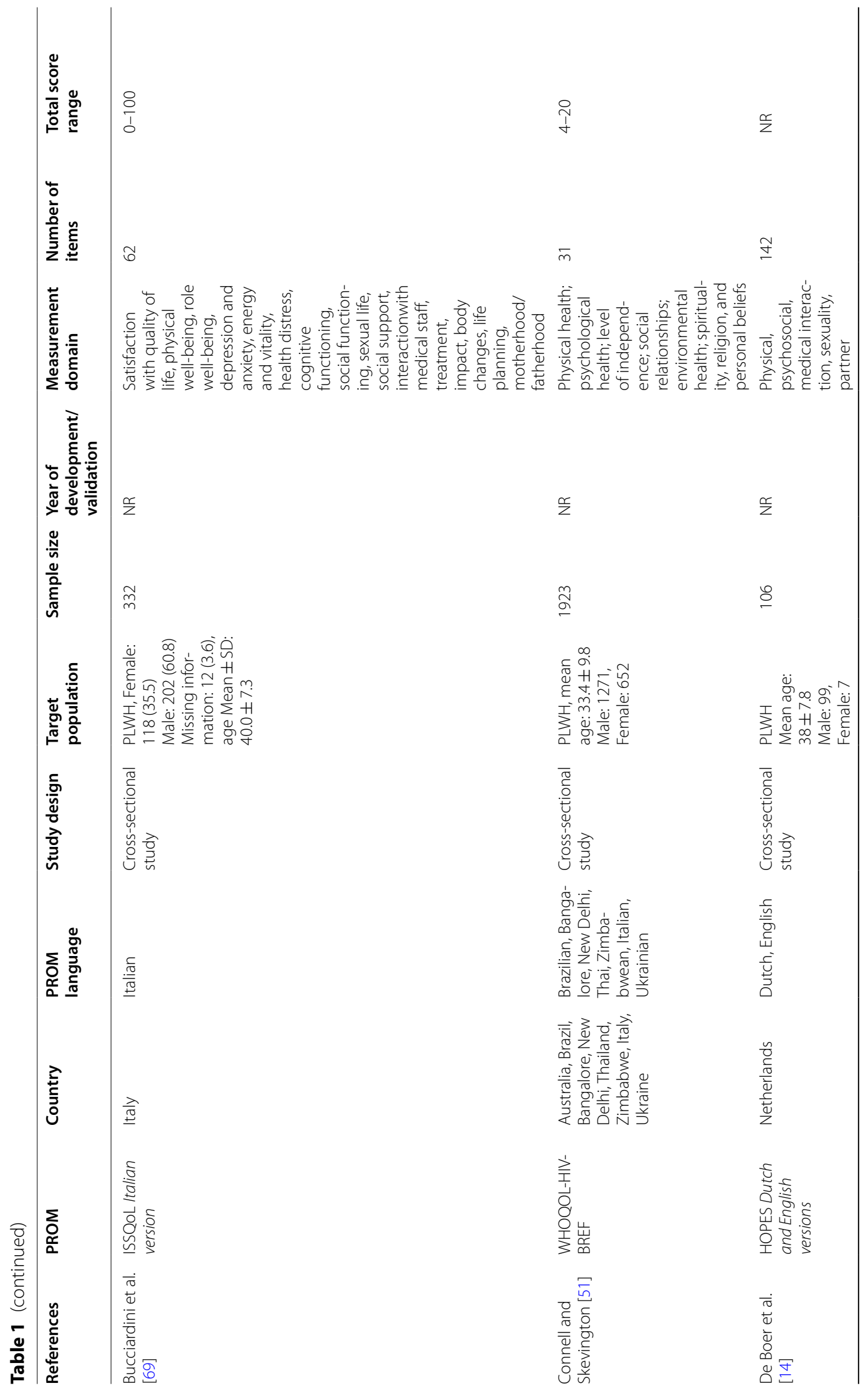




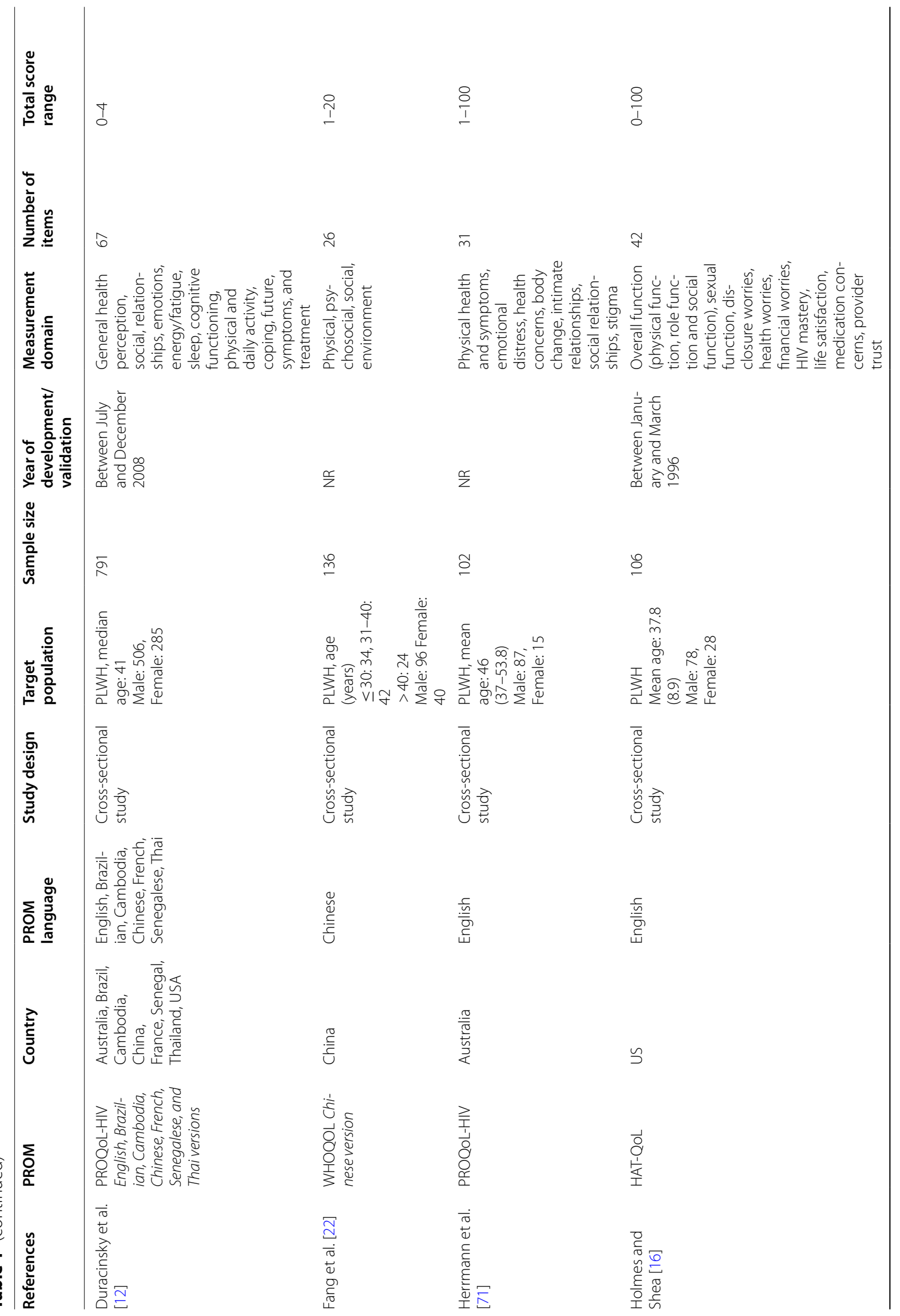




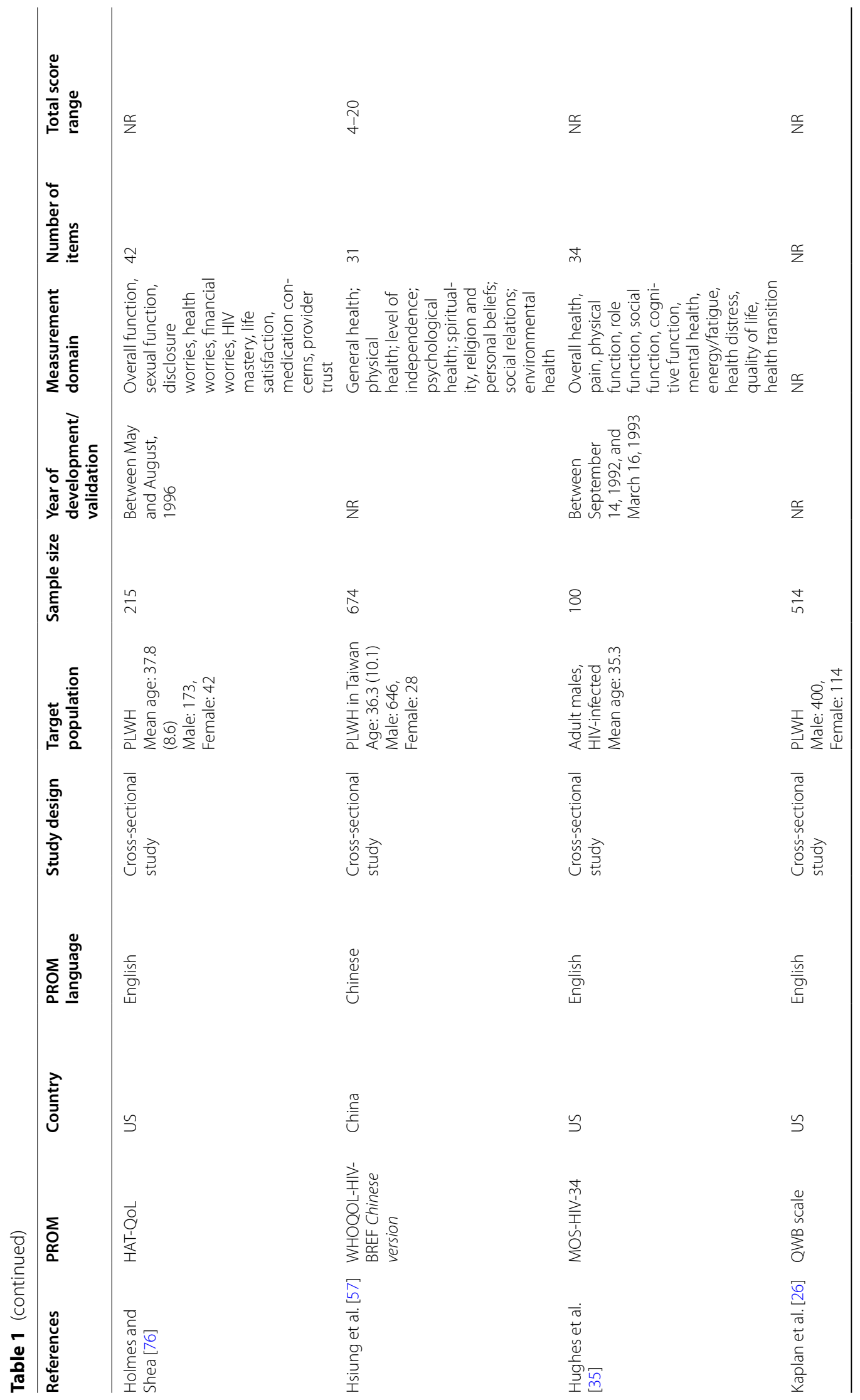




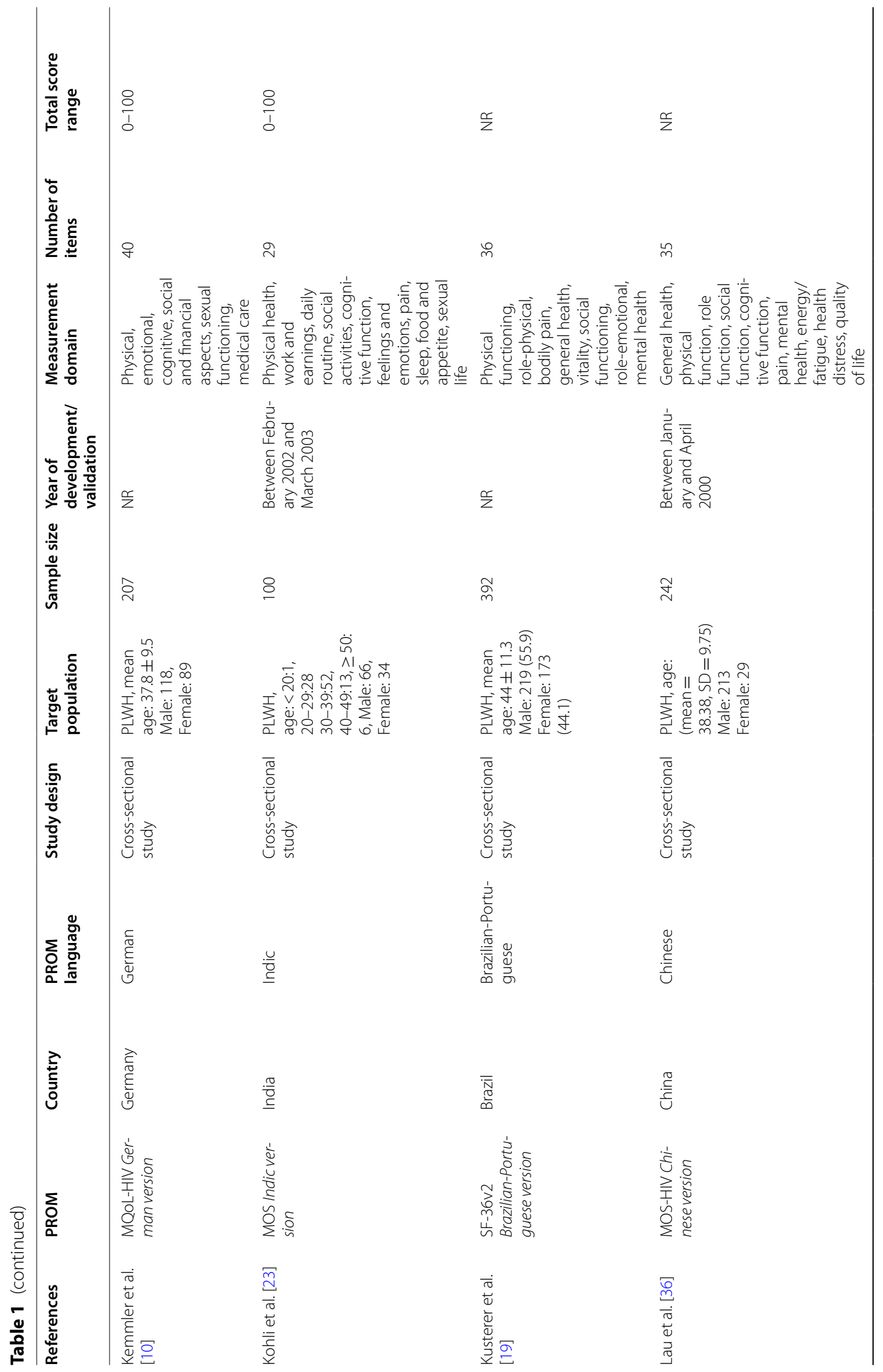




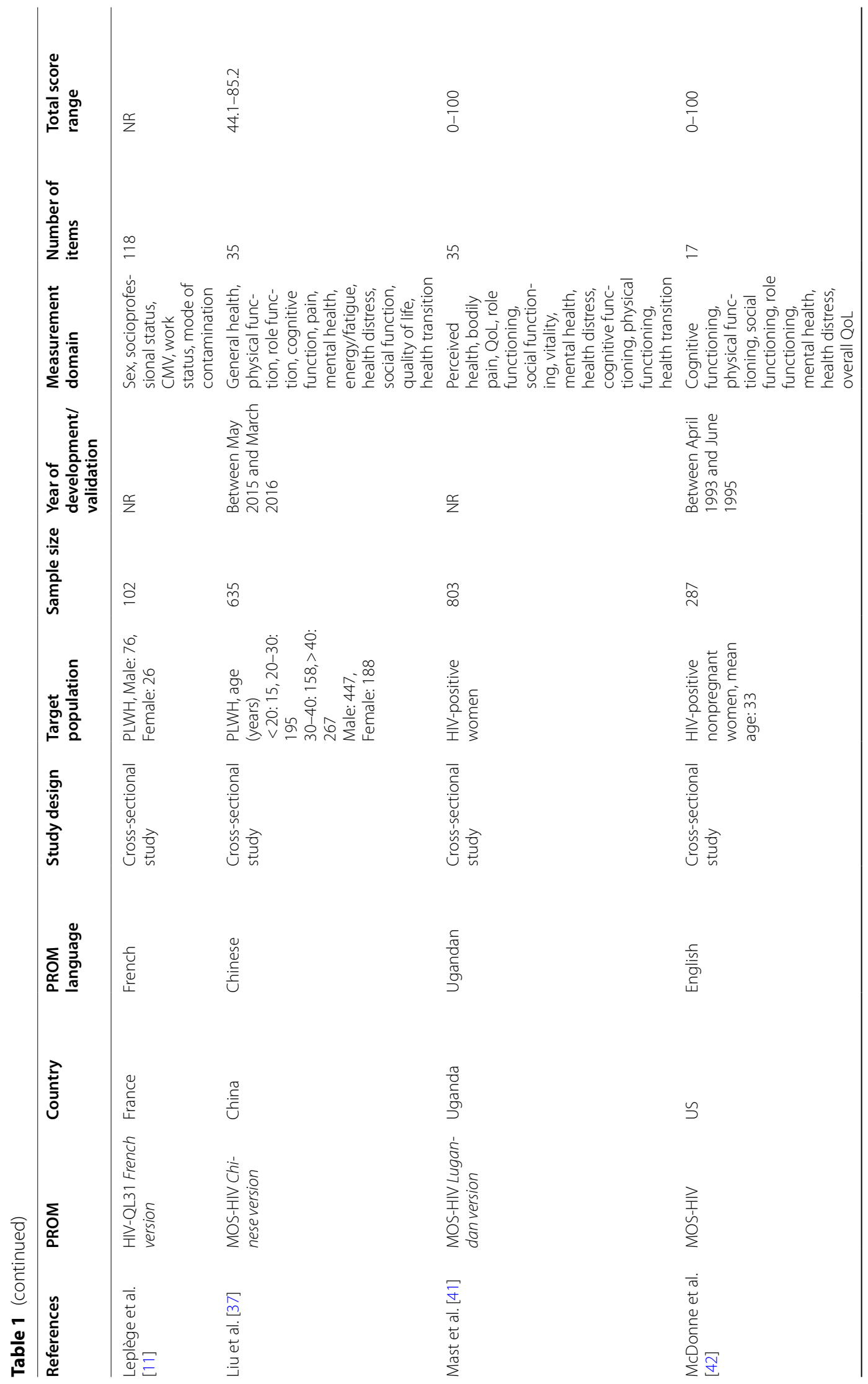




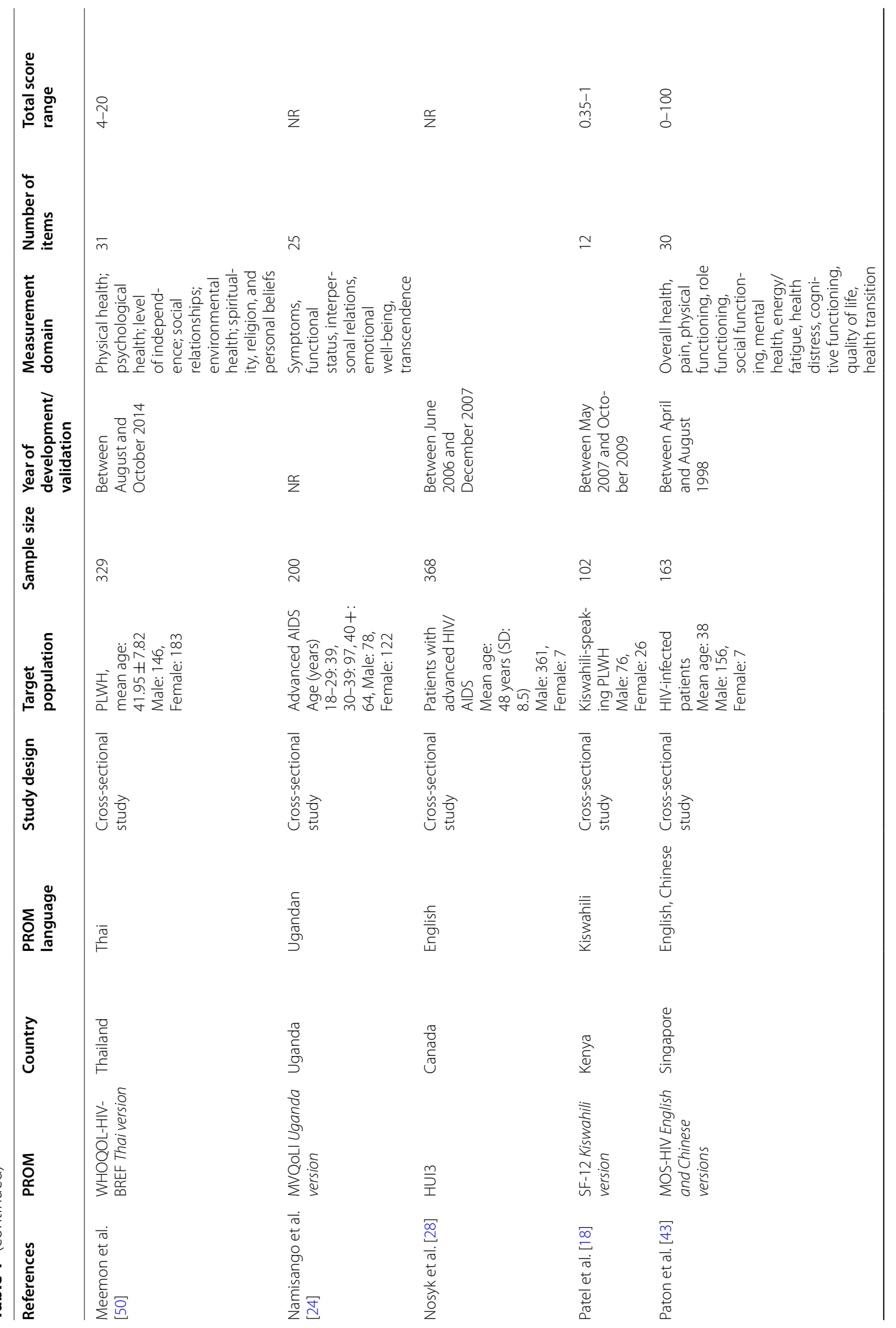




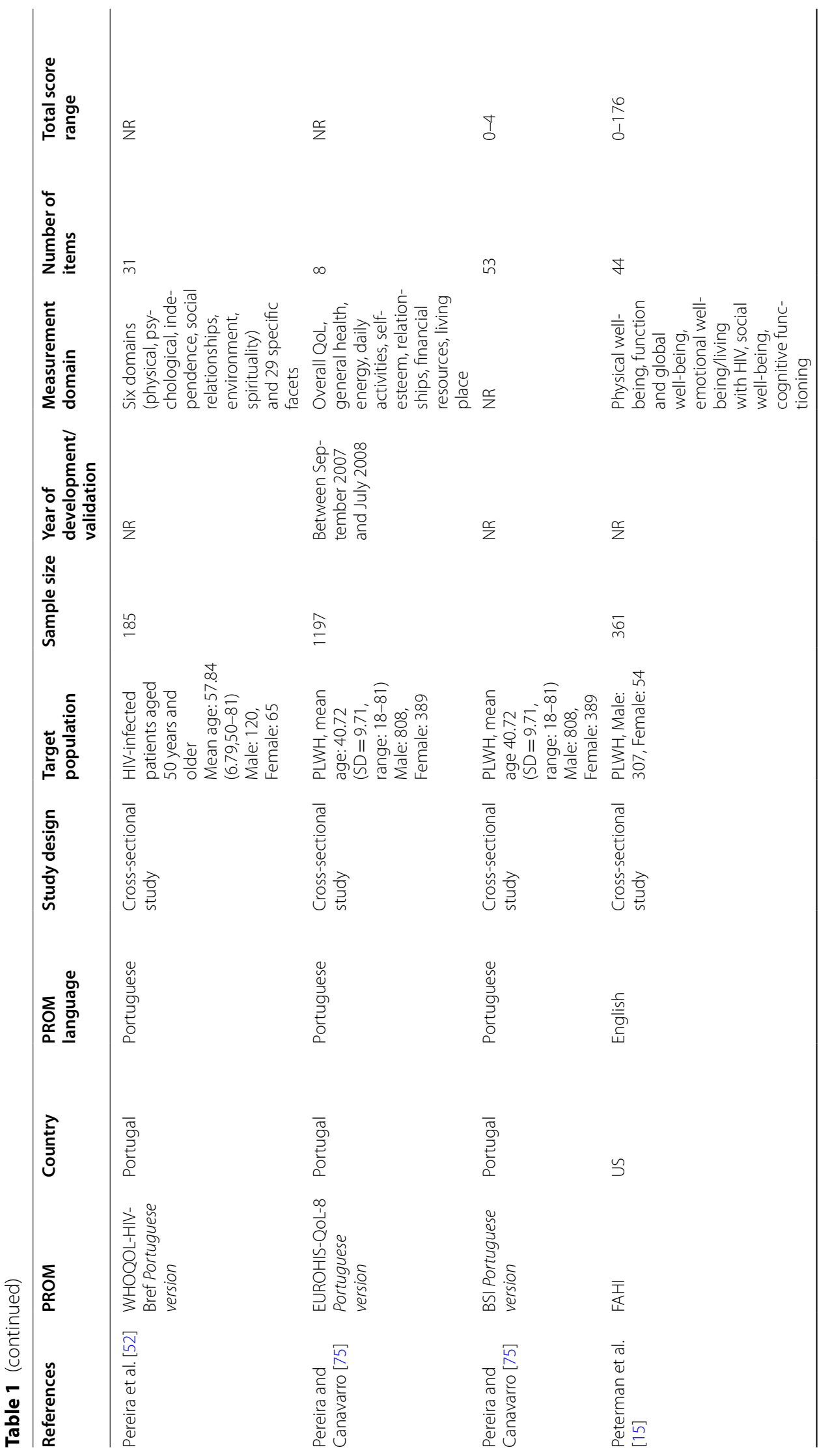




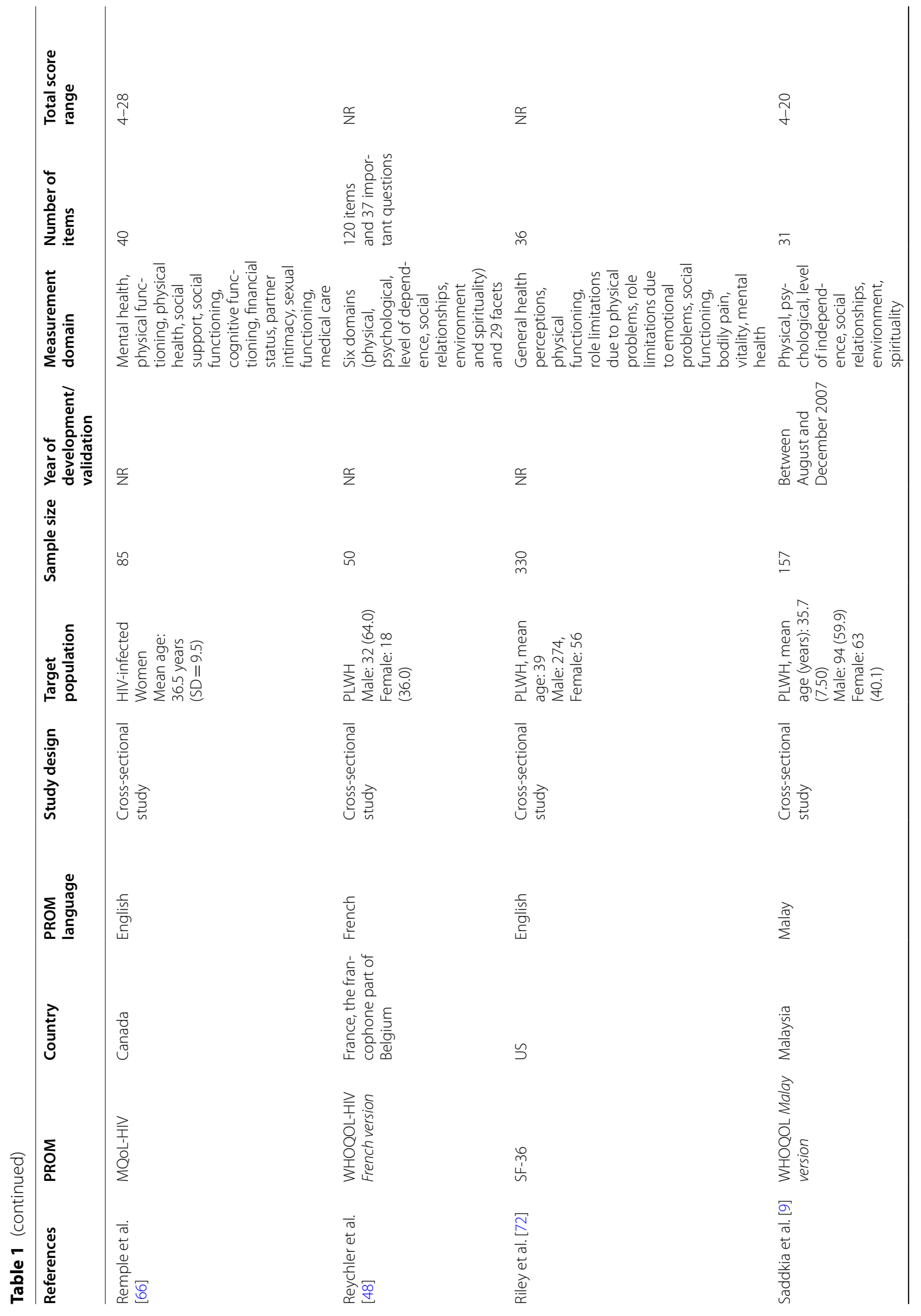




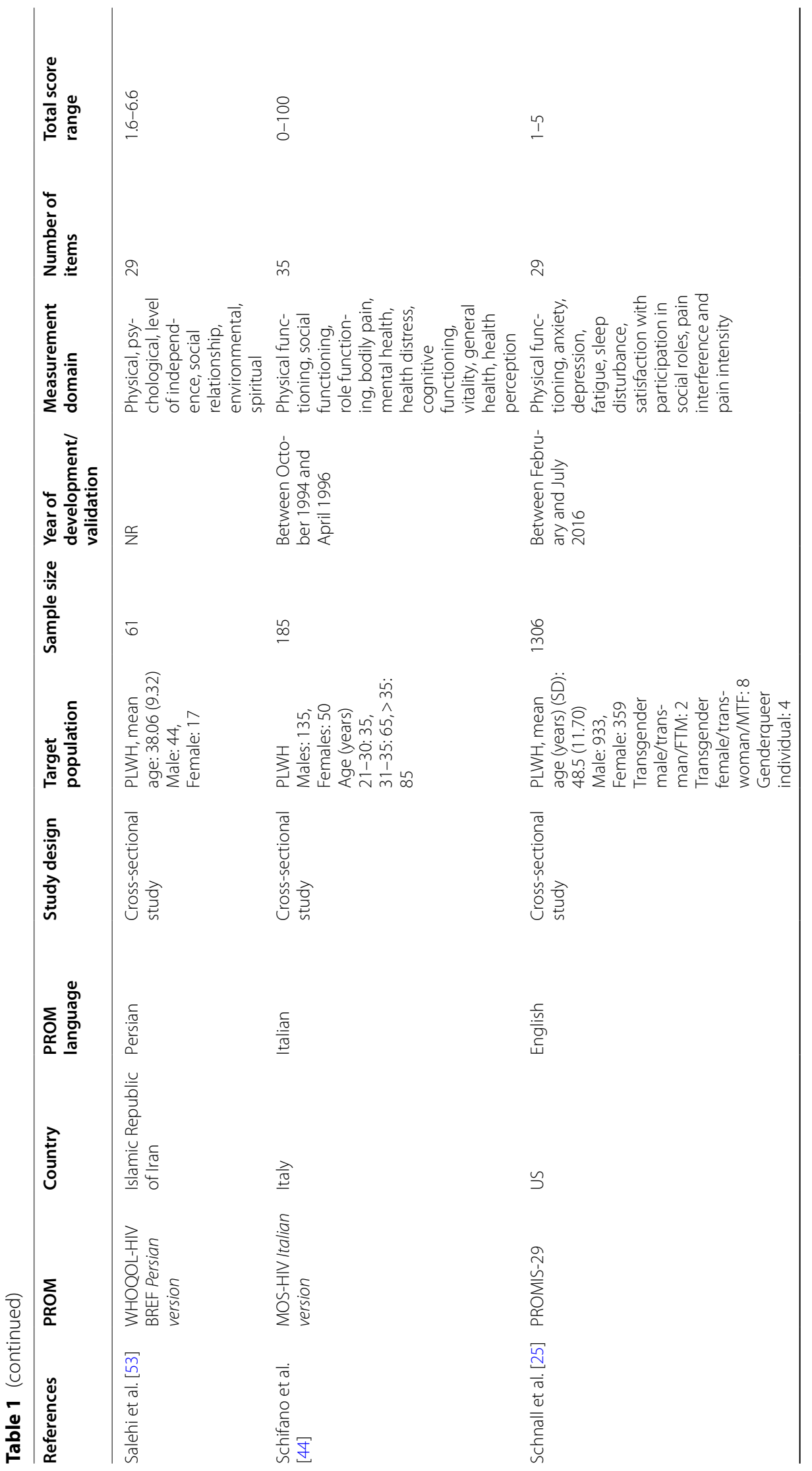




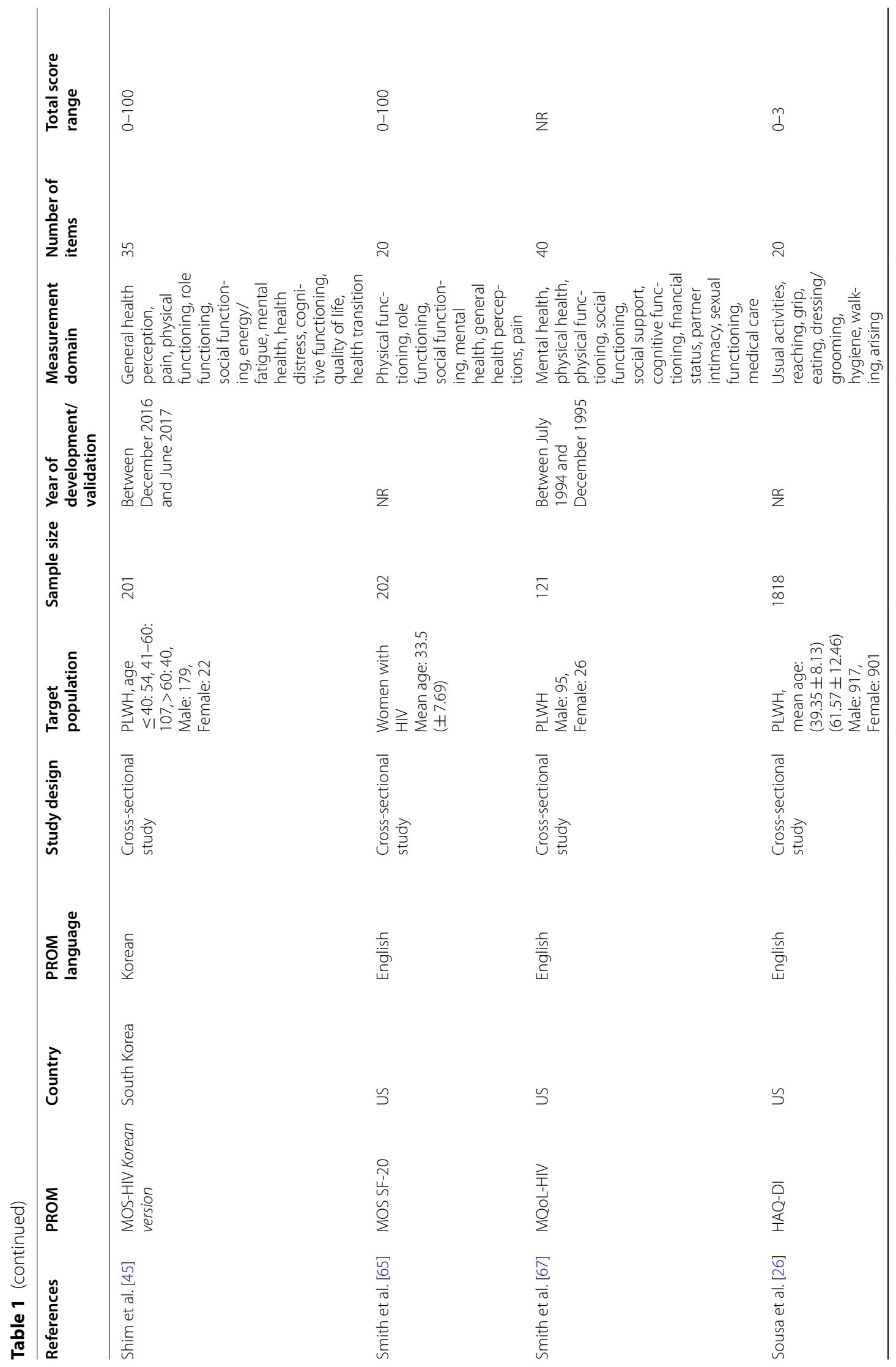




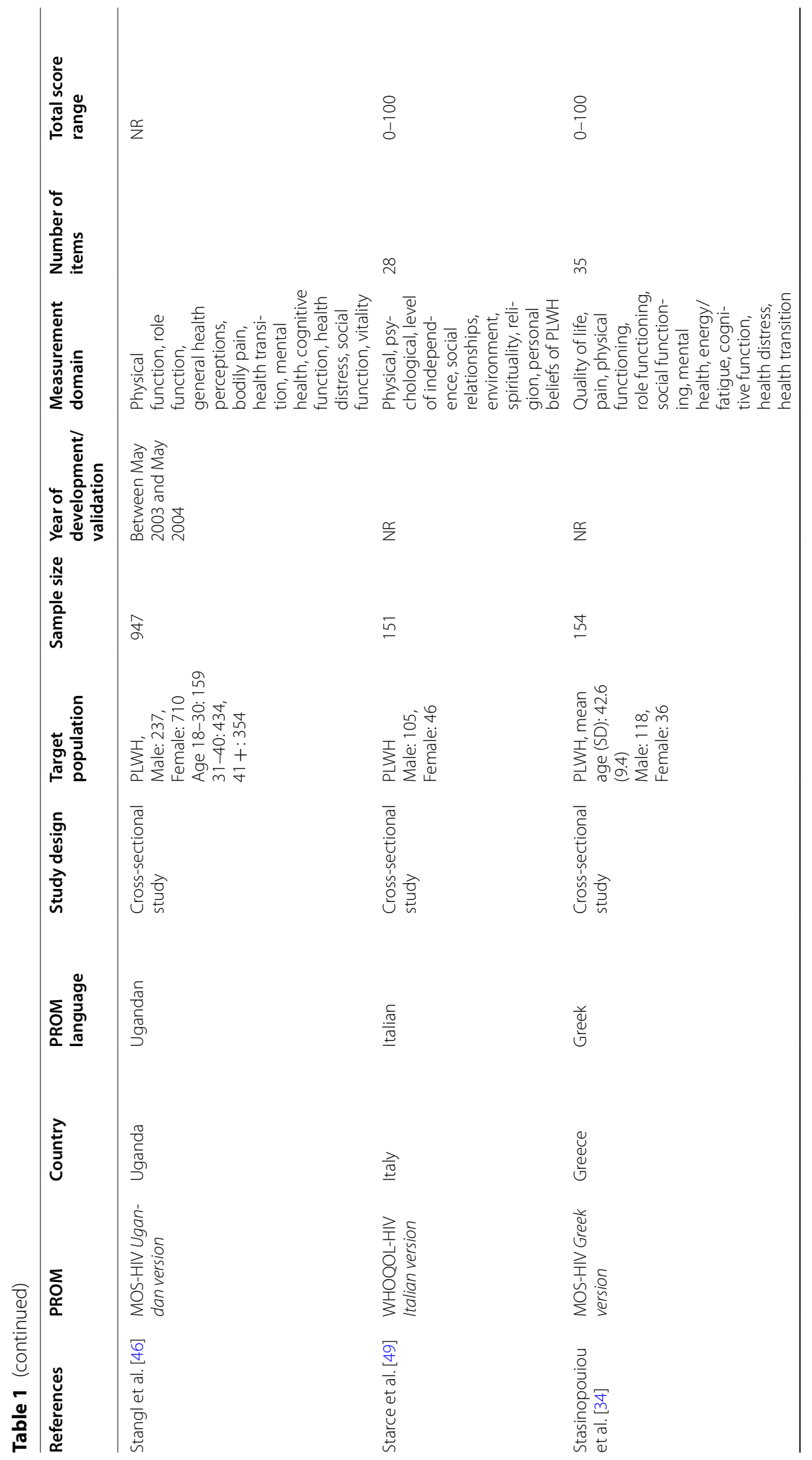




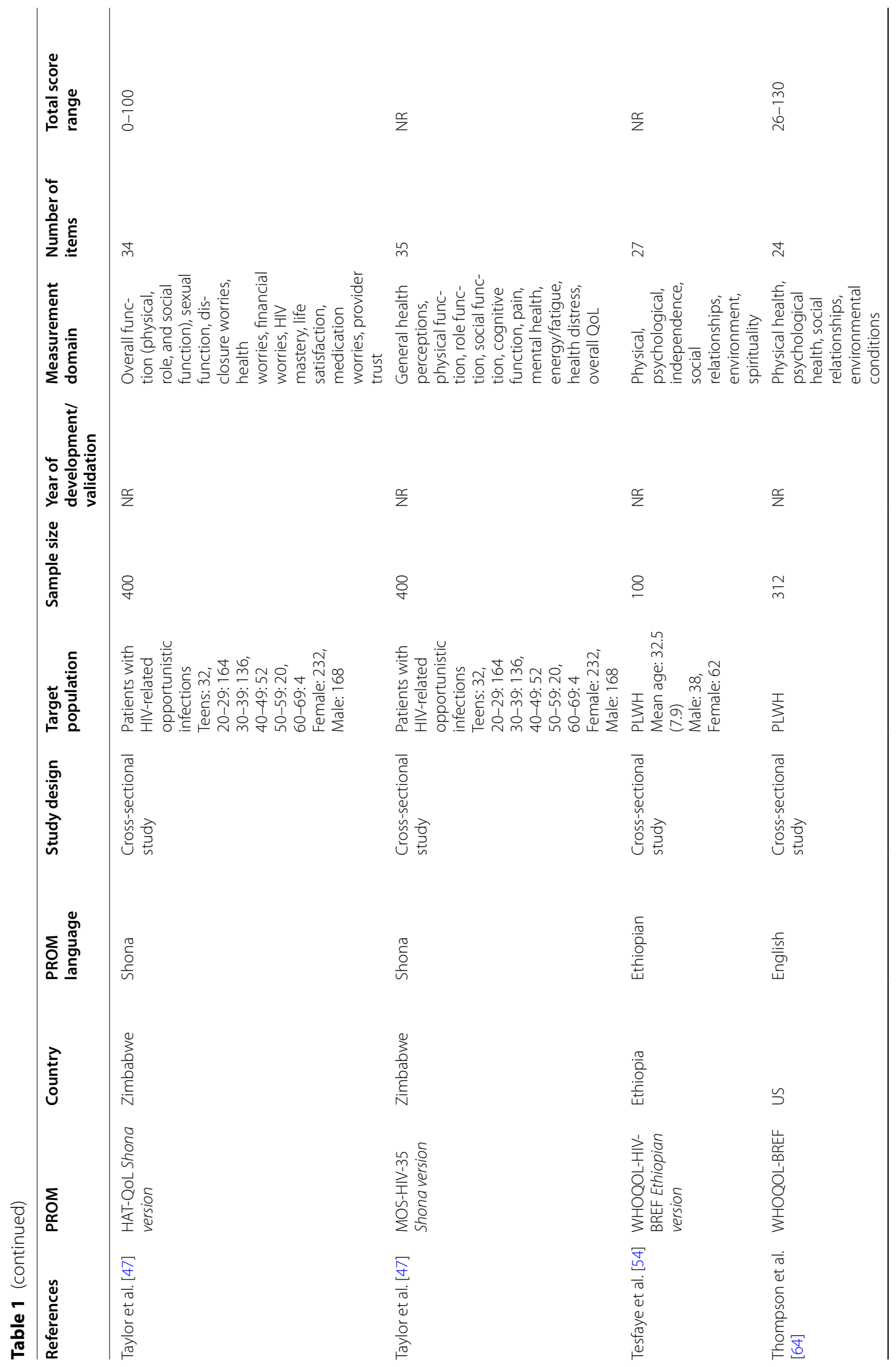




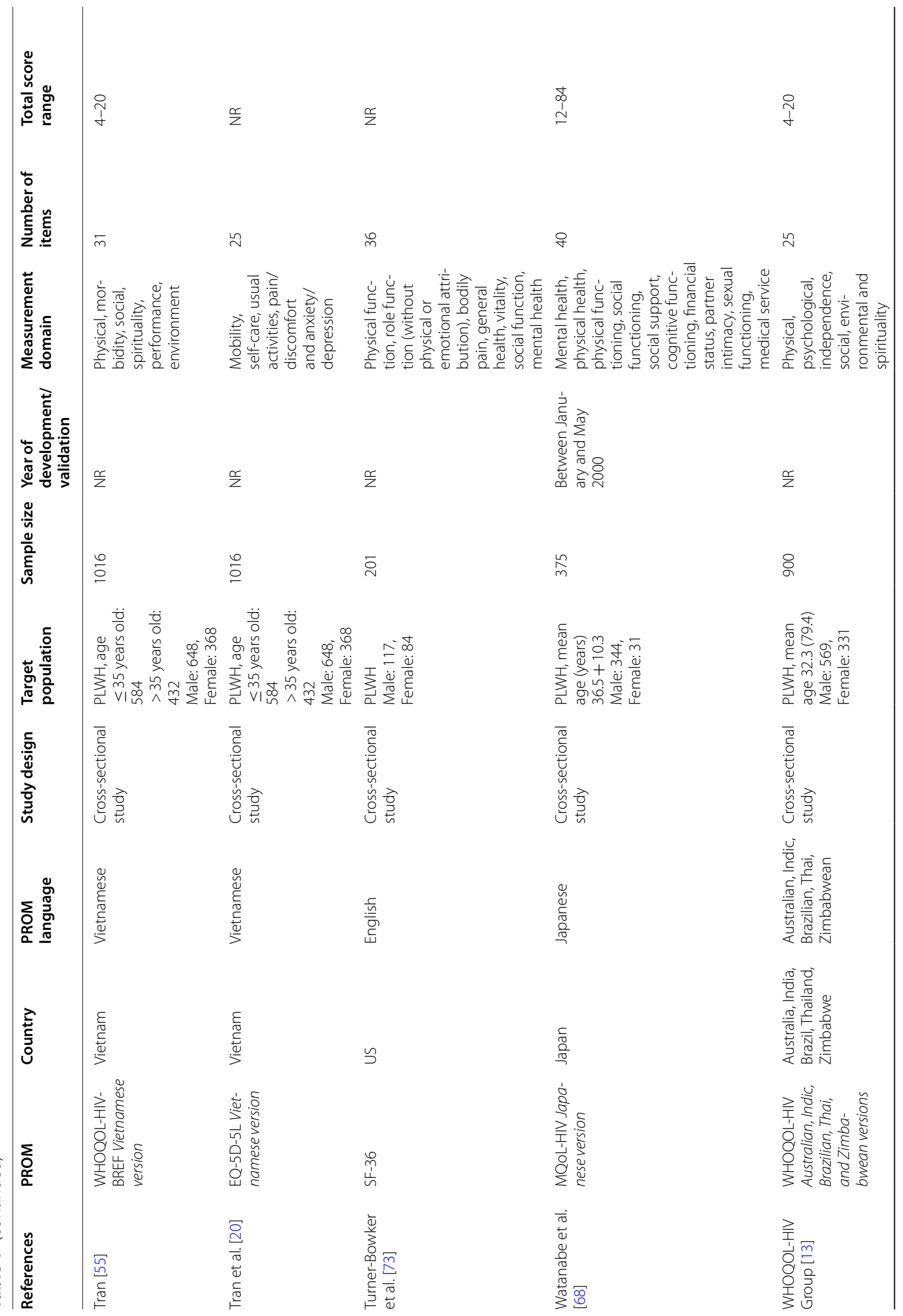




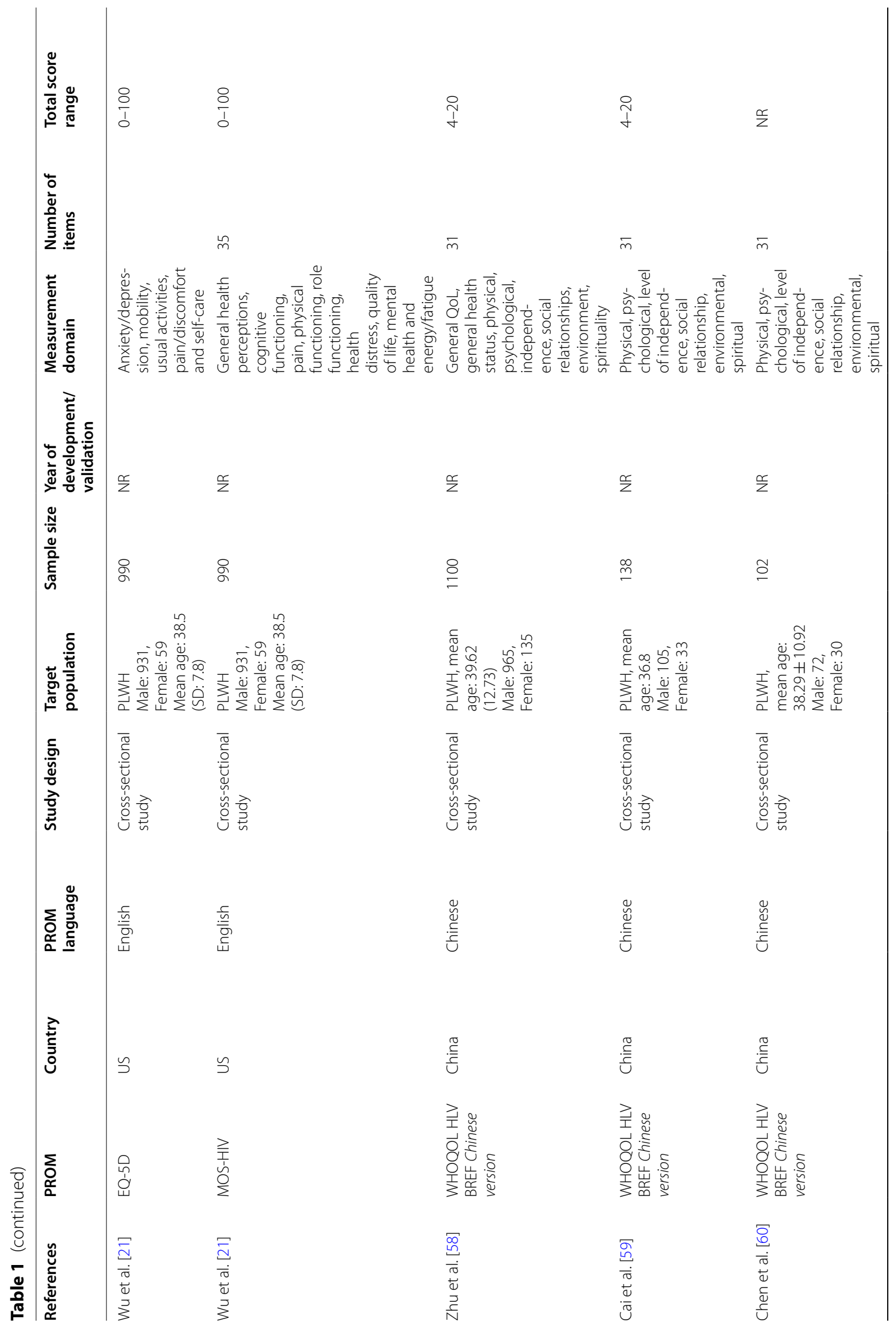




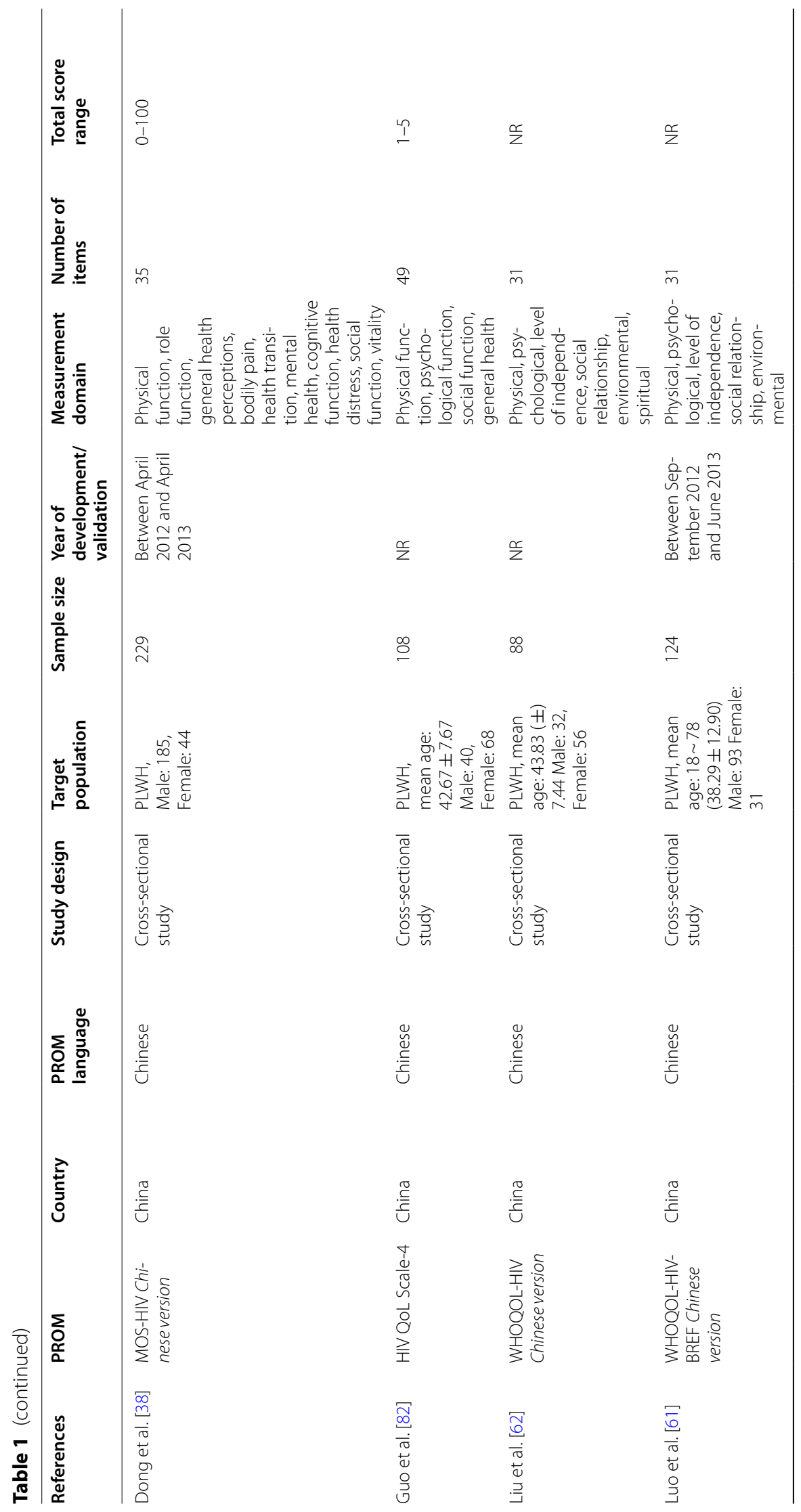




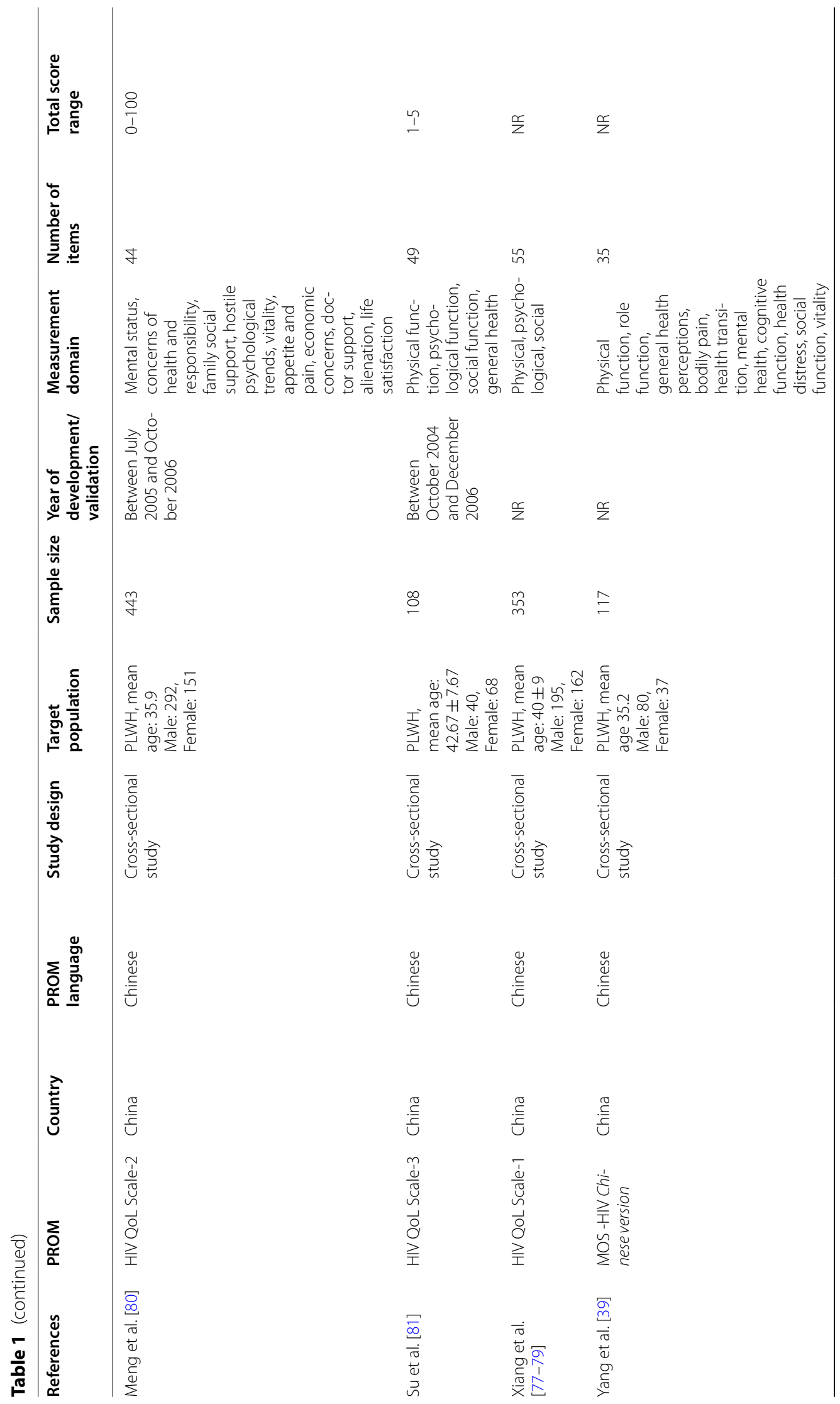




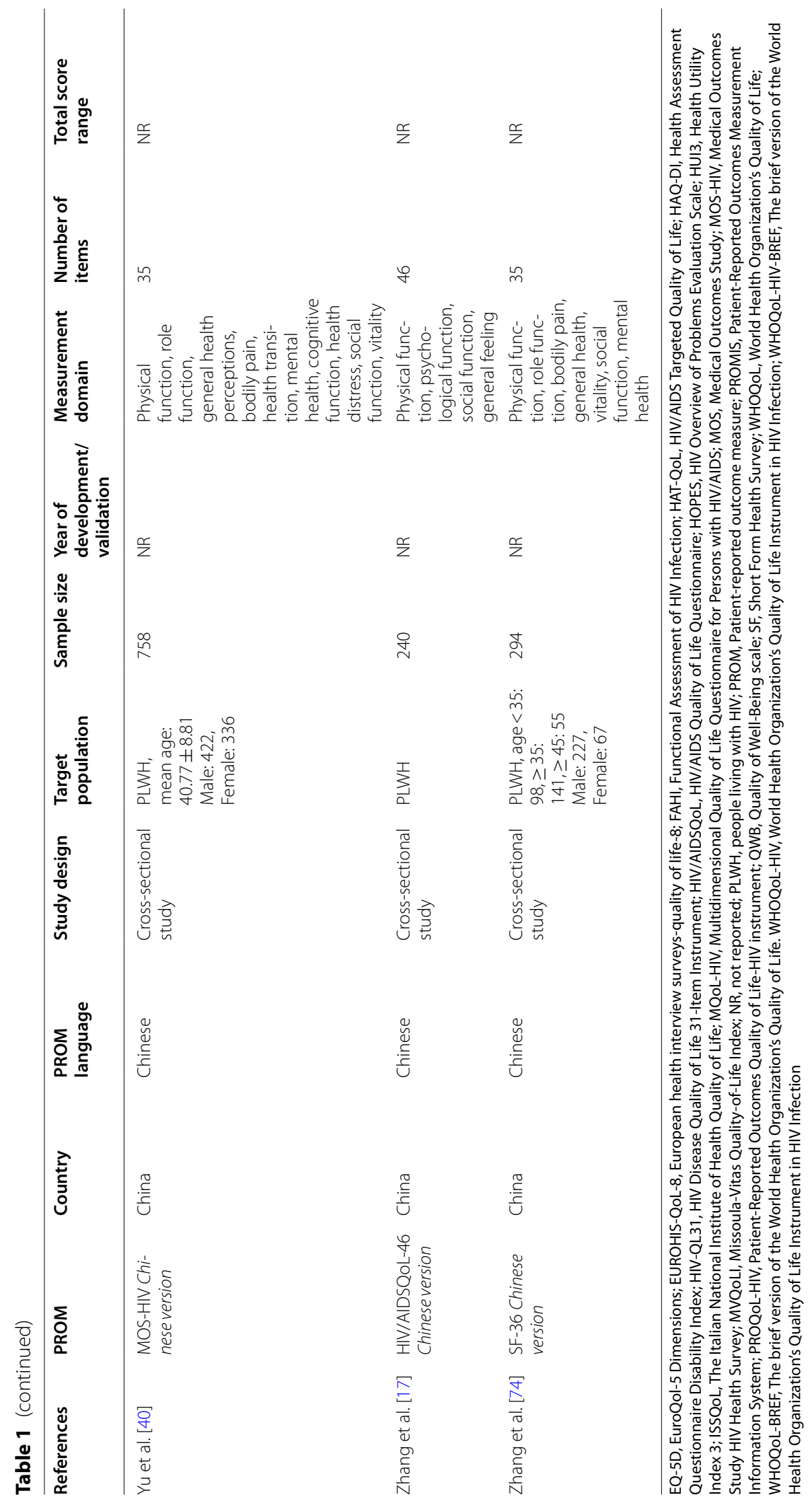


[52, 75], and two in Canada [28, 66]. A total of 28,480 participants were included, with sample sizes ranging from 50 to 1923 [9-28, 34-82]. One study was conducted with adult males [35]. Four studies were conducted with HIV-positive women $[41,42,65,66]$. One study was conducted with HIV-infected patients aged 50 years and older [52], and two studies were conducted with people with advanced AIDS [24, 28]. One study involved transgender male, transgender female, and genderqueer individuals [25]. One study was conducted in patients with HIV-related opportunistic infections [47].

The characteristics of all 30 HRQoL PROMs, including the items, domains, and score range, are shown in Table 1. The total number of items ranged from 8 to 142 [9-28, 34-82]. A total of 10 PROMs had multiple language versions, and the remaining 18 had only one language version. Tables 4 and 5 summarize the psychometric properties of the HIV-specific and generic instruments.

\section{Quality assessment}

\section{Methodological quality assessment}

Tables 2 and 3 show the methodological quality of the 69 included studies based on the COSMIN checklist. All studies were considered to have sufficient methodological quality for further study. Table 2 presents an overview of the COSMIN ratings of the HIV-specific instruments, and Table 3 presents the generic instruments. Limited information was retrieved on cross cultural validity/ translation (58 studies) [11-14, 16-23, 25-28, 35-40, $42-44,47,48,50-56,58-64,66-82]$, criterion validity (59 studies) [9-12, 15-17, 19-26, 34, 37-50, 52-67, 69$79,81,82]$, reliability (49 studies) [11, 13-21, 23-28, 34$36,38,39,41-47,49-55,57,59,62-65,68,69,72-76]$, hypothesis testing (18 studies) [11, 16, 17, 34, 38, 39, 41, $53,61,67,68,71,77-82]$ and responsiveness (62 studies) [9-16, 18-20, 22-27, 34-45, 47-57, 59-64, 66, 68-82]. No data were identified on error and interpretability.

\section{Quality of measurement properties of assessments}

Table 4 presents the quality of the psychometric properties retrieved from the 69 included studies for all 30 measures. Fifteen PROMs were rated as insufficient (-) for content validity $[11,17,48,49,53,57,59-61,77-82]$. There were 19 PROMs $[19,24,26,37-40,45,51-54,57$, $59,60,64,70,74,75]$ rated as sufficient $(+)$ for construct validity, and 31 [10-12, 14-17, 21, 34-36, 41, 42, 44, 46$48,50,53,55,56,58,59,67,68,76-79,81,82]$ were rated as insufficient $(-)$. The internal consistency was rated as sufficient (+) for 59 PROMs [9-19, 22-25, 34, 36-49, $51-72,74,76-82]$ and as insufficient (-) for 4 PROMs $[20,21,35,50]$.

\section{Certainty of evidence}

Table 5 shows the overall quality score for each measurement property of the HIV-specific and generic instruments. Five PROMs were strongly recommended based on the methodological quality of each psychometric property, including MOS-HIV, WHOQoL-HIV-BREF, SF-36, MQoL-HIV, and WHOQoL-HIV. Among the seven language versions of the MOS-HIV [21, 34-47], six were rated as "high" for internal consistency [21, 34, $35,41-47]$, and one was rated as "moderate" [36-40]. There were three versions rated as "high" for cross-cultural validity/translation [34, 41, 44, 46]. Among the eight versions of the WHOQoL-HIV-BREF [50-61], five were rated as "high" for internal consistency [50-52, 54, 56], and one was rated as "moderate" [53]. In total, more studies of the MOS-HIV were rated as "high" than studies of the WHOQoL-HIV-BREF, and more studies of the WHOQoL-HIV-BREF were rated as "very low" than studies of the MOS-HIV.

\section{Discussion}

This systematic review identified and assessed the psychometric properties of $30 \mathrm{HRQoL}$ PROMs in PLWH and evaluated the certainty of the evidence provided for each PROM. To the best of our knowledge, this is the first and most comprehensive systematic review summarizing all psychometric properties of HRQoL PROMs for PLWH. The results may provide quantitative evidence for researchers and healthcare professionals to choose PROMs measuring HRQoL in PLWH in future scientific research and clinical practice.

Our systematic review found that compared to other HIV-specific and generic PROMs, the MOS-HIV has the best psychometric properties. The MOS-HIV is the most widely used HIV-specific instrument. In total, we searched fourteen validation studies to evaluate the psychometric properties of eight different language versions of MOS-HIV. Chinese included both simplified and traditional versions. Only one version was rated as "moderate" in internal consistency, and the other was rated as "high". The MOS-HIV also has good construct validity, criterion validity, and hypothesis testing for construct validity. Overall, the expert group classified MOS-HIV as strongly recommended based on the GRADE system. Our results were in line with previous studies. Cooper and colleagues conducted umbrella reviews and found that the MOS-HIV was also recommended as a suitable measure for assessing HRQoL in PLWH from a content perspective [29]. In general, the MOS-HIV was considered to have good psychometric properties. Good internal consistency was generally reported, and its reliability was considered adequate [83, 84]. Acceptable convergent 


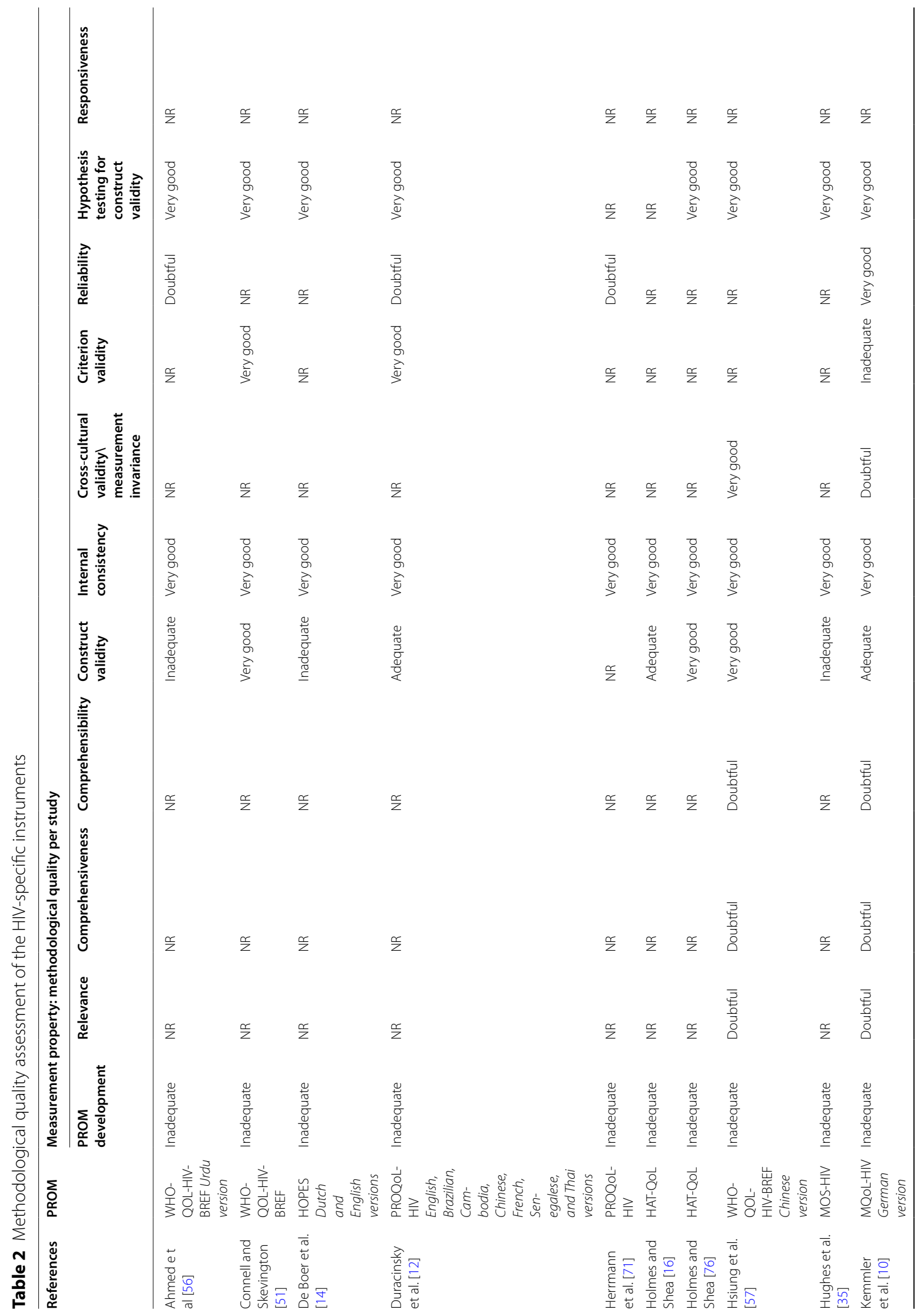




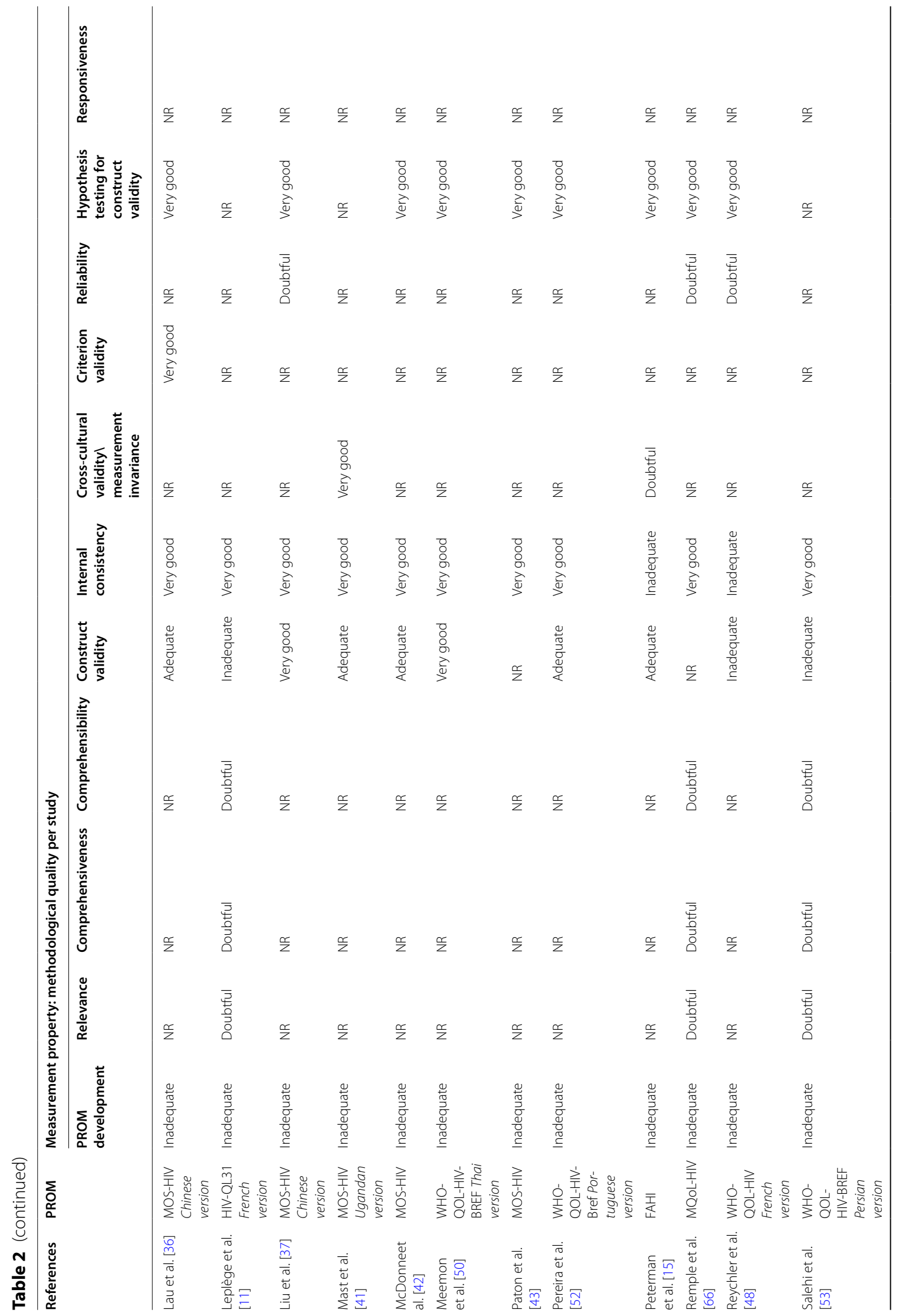




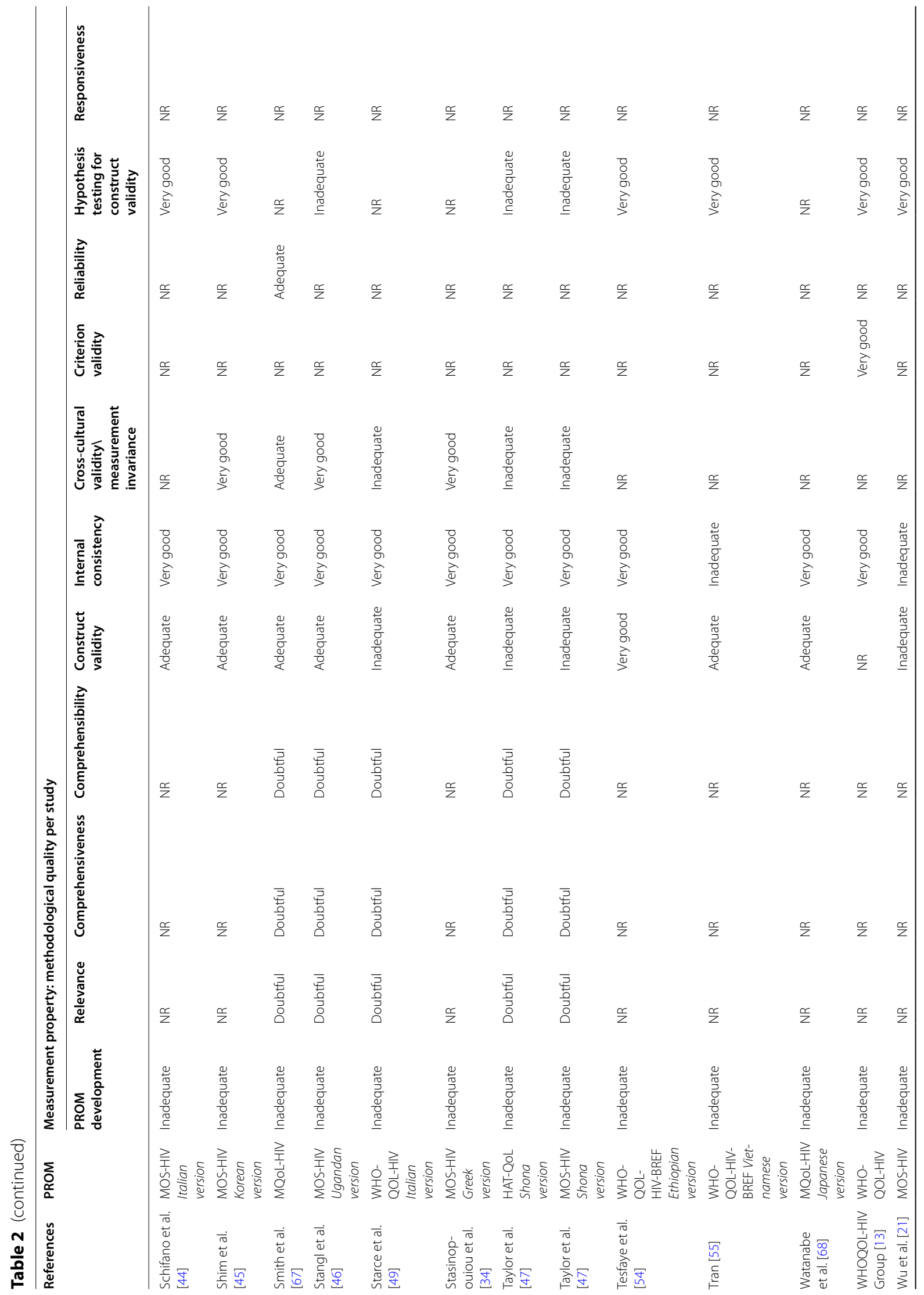




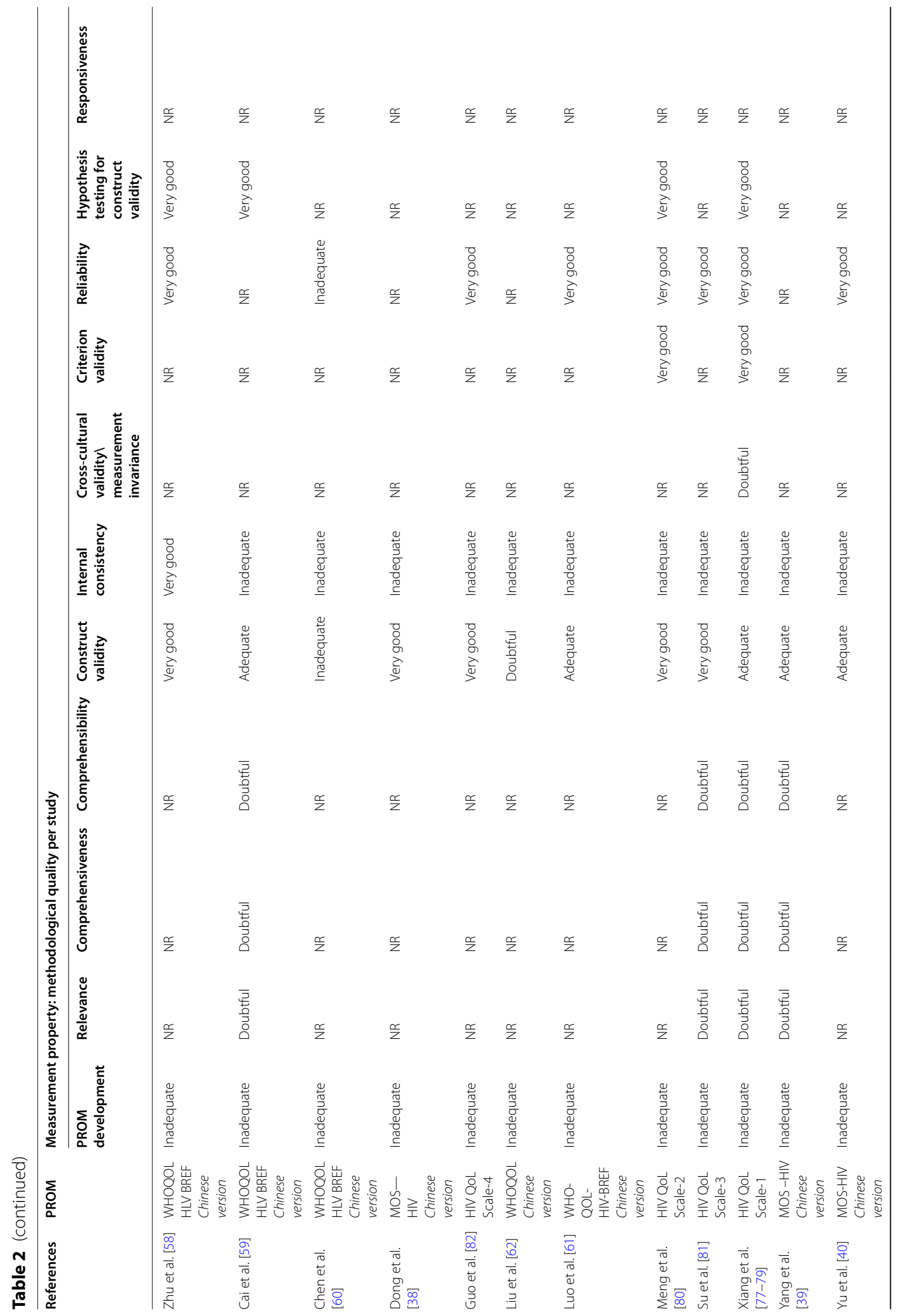




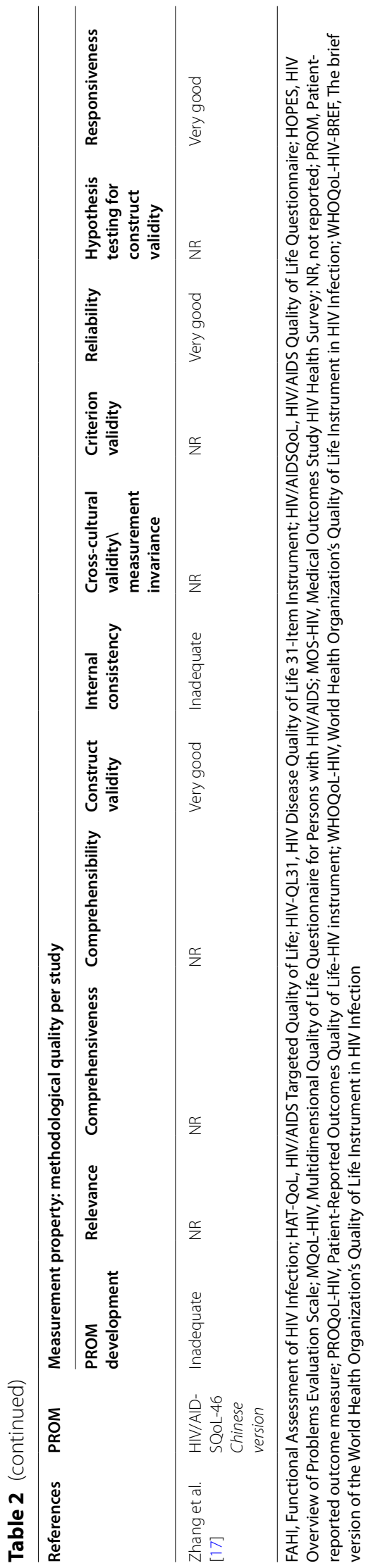




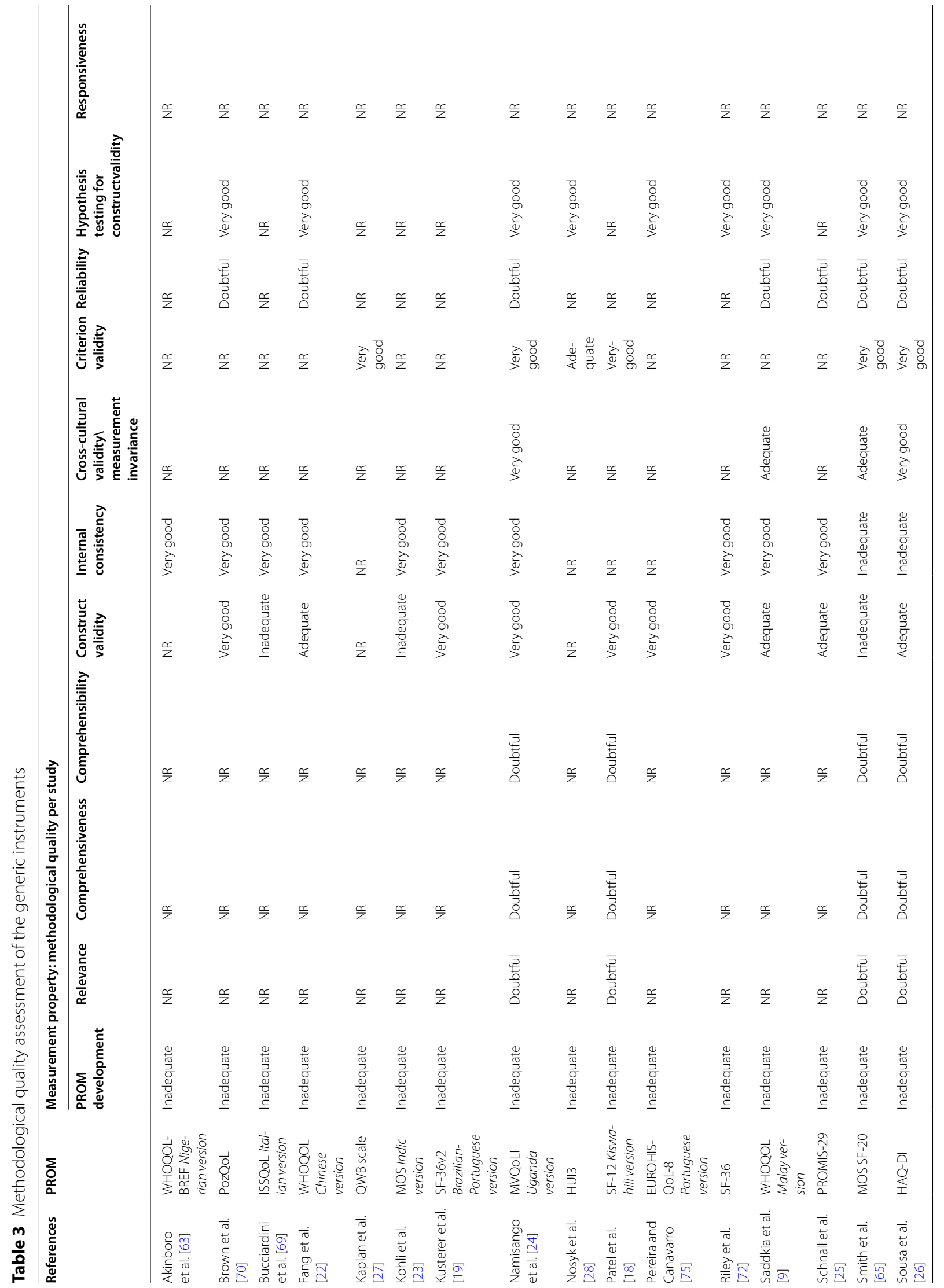




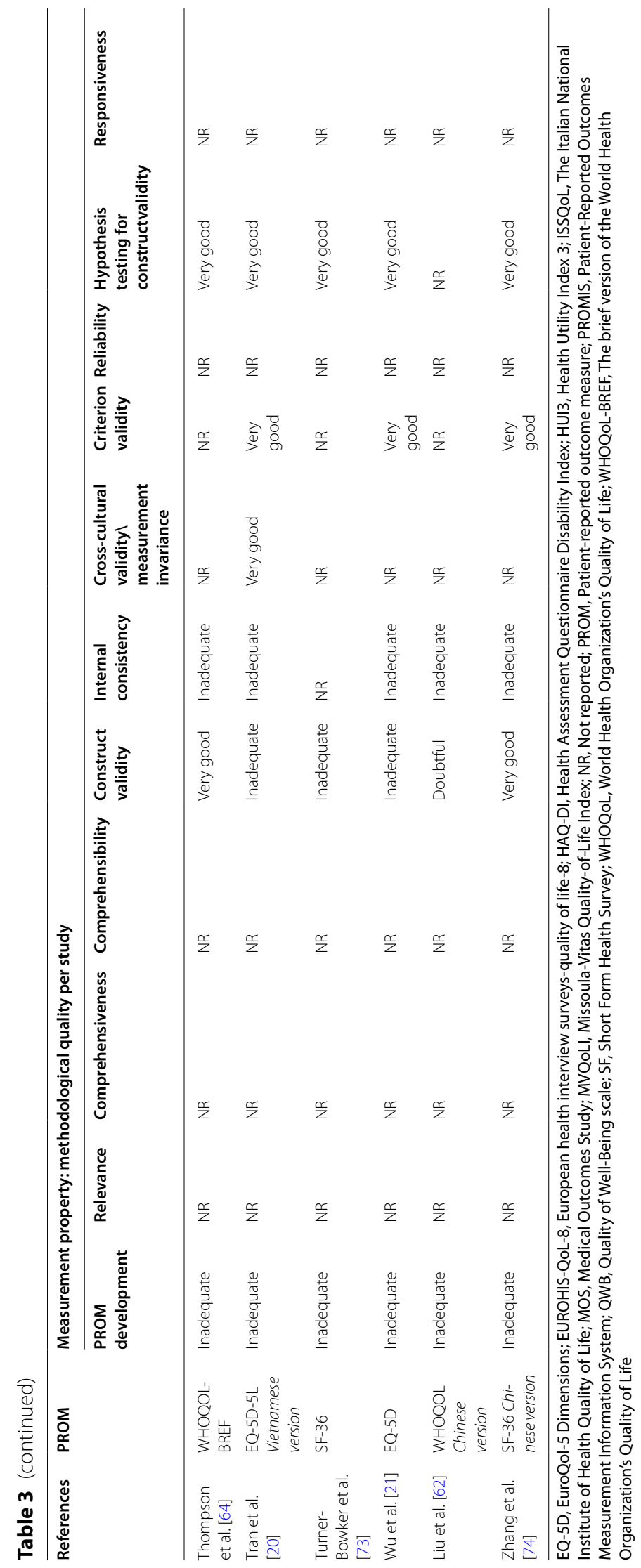


Table 4 Rating of the measurement properties of the instruments

\begin{tabular}{|c|c|c|c|c|c|c|c|c|c|}
\hline PROM & References & $\begin{array}{l}\text { Construct } \\
\text { validity } \\
\text { (CFI) }\end{array}$ & $\begin{array}{l}\text { Internal } \\
\text { consistency } \\
\text { (Cronbach's } \\
\text { alpha) }\end{array}$ & $\begin{array}{l}\text { Reliability } \\
\text { (ICC) }\end{array}$ & $\begin{array}{l}\text { Measurement } \\
\text { error }\end{array}$ & $\begin{array}{l}\text { Hypothesis } \\
\text { testing for } \\
\text { construct } \\
\text { validity }\end{array}$ & $\begin{array}{l}\text { Cross-cultural } \\
\text { validity } \ \\
\text { measurement } \\
\text { invariance }\end{array}$ & $\begin{array}{l}\text { Criterion } \\
\text { validity }\end{array}$ & Responsiveness \\
\hline $\begin{array}{l}\text { WHOQOL- } \\
\text { HIV }\end{array}$ & $\begin{array}{l}\text { WHOQOL- } \\
\text { HIV Group } \\
{[13]}\end{array}$ & NR & $+(0.87-0.94)$ & $N R$ & NR & $?$ & $N R$ & - & NR \\
\hline $\begin{array}{l}\text { WHOQOL- } \\
\text { HIV French } \\
\text { version }\end{array}$ & $\begin{array}{l}\text { Reychler } \\
\text { et al. [48] }\end{array}$ & - & $+(0.94)$ & $\begin{array}{l}-(0.42- \\
0.74)\end{array}$ & NR & $?$ & NR & NR & NR \\
\hline $\begin{array}{l}\text { WHOQOL- } \\
\text { HIV Italian } \\
\text { version }\end{array}$ & $\begin{array}{l}\text { Starce et al. } \\
\text { [49] }\end{array}$ & NR & $+(0.53-0.89)$ & NR & NR & $?$ & + & NR & NR \\
\hline $\begin{array}{l}\text { WHOQOL- } \\
\text { HIV-BREF }\end{array}$ & $\begin{array}{l}\text { Connell and } \\
\text { Skevington } \\
{[51]}\end{array}$ & $+(0.97)$ & $+(0.74-0.82)$ & NR & NR & + & NR & - & NR \\
\hline $\begin{array}{l}\text { WHOQOL- } \\
\text { HIV-BREF } \\
\text { Thai version }\end{array}$ & $\begin{array}{l}\text { Meemon } \\
\text { et al. [50] }\end{array}$ & - & $+(0.91)$ & NR & NR & + & NR & NR & NR \\
\hline $\begin{array}{l}\text { WHOQOL- } \\
\text { HIV-BREF } \\
\text { Portuguese } \\
\text { version }\end{array}$ & $\begin{array}{l}\text { Pereira et al. } \\
{[52]}\end{array}$ & $+(0.97)$ & $+(0.65-0.86)$ & NR & NR & + & NR & NR & NR \\
\hline $\begin{array}{l}\text { WHOQOL- } \\
\text { HIV BREF } \\
\text { Persian } \\
\text { version }\end{array}$ & $\begin{array}{l}\text { Salehi et al. } \\
\text { [53] }\end{array}$ & - & $+(0.87)$ & NR & NR & NR & NR & NR & NR \\
\hline $\begin{array}{l}\text { WHOQOL- } \\
\text { HIV-BREF } \\
\text { Ethiopian } \\
\text { version }\end{array}$ & $\begin{array}{l}\text { Tesfaye } \\
\text { et al. [54] }\end{array}$ & $+(0.82)$ & $+(0.93)$ & NR & NR & + & NR & NR & NR \\
\hline $\begin{array}{l}\text { WHOQOL- } \\
\text { HIV-BREF } \\
\text { Vietnamese } \\
\text { version }\end{array}$ & Tran [55] & - & $+(0.67-0.89)$ & NR & NR & $?$ & NR & NR & NR \\
\hline $\begin{array}{l}\text { WHOQOL- } \\
\text { HIV-BREF } \\
\text { Urdu version }\end{array}$ & $\begin{array}{l}\text { Ahmed } \\
\text { et al. [56] }\end{array}$ & - & $+(0.93)$ & $\begin{array}{l}+(0.87- \\
0.99)\end{array}$ & NR & $?$ & NR & NR & NR \\
\hline \multirow{5}{*}{$\begin{array}{l}\text { WHOQOL- } \\
\text { HIV-BREF } \\
\text { Chinese } \\
\text { version }\end{array}$} & $\begin{array}{l}\text { Hsiung } \\
\text { et al. [57] }\end{array}$ & $+(0.95)$ & $+(0.67-0.80)$ & NR & NR & + & + & NR & NR \\
\hline & $\begin{array}{l}\text { Zhu et al. } \\
\text { [58] }\end{array}$ & $-(0.81)$ & $+(0.93)$ & $\begin{array}{l}+(0.72- \\
0.82)\end{array}$ & NR & + & NR & NR & + \\
\hline & $\begin{array}{l}\text { Cai et al. } \\
\text { [59] }\end{array}$ & - & $+(0.60-0.82)$ & NR & NR & + & NR & NR & NR \\
\hline & $\begin{array}{l}\text { Chen et al. } \\
\text { [60] }\end{array}$ & - & $+(>0.60)$ & $-(>0.50)$ & NR & + & NR & NR & NR \\
\hline & $\begin{array}{l}\text { Luo et al. } \\
\text { [61] }\end{array}$ & - & $+(0.60-0.76)$ & $\begin{array}{l}+(0.47- \\
0.68)\end{array}$ & NR & NR & NR & NR & NR \\
\hline \multirow[t]{4}{*}{ MOS-HIV } & $\begin{array}{l}\text { Hughes } \\
\text { et al. [35] }\end{array}$ & - & $-(0.57-0.89)$ & NR & NR & + & NR & - & NR \\
\hline & $\begin{array}{l}\text { McDone } \\
\text { et al. [42] }\end{array}$ & - & $+(0.64-0.89)$ & NR & NR & $?$ & NR & NR & NR \\
\hline & $\begin{array}{l}\text { Paton et al. } \\
\text { [43] }\end{array}$ & NR & $+(>0.70)$ & NR & NR & + & NR & NR & NR \\
\hline & $\begin{array}{l}\text { Wu et al. } \\
{[21]}\end{array}$ & - & - & NR & NR & $?$ & NR & NR & + \\
\hline $\begin{array}{l}\text { MOS-HIV } \\
\text { Greek ver- } \\
\text { sion }\end{array}$ & $\begin{array}{l}\text { Stasinopo } \\
\text { et al. [34] }\end{array}$ & - & $+(>0.80)$ & NR & NR & NR & + & NR & NR \\
\hline
\end{tabular}


Table 4 (continued)

\begin{tabular}{|c|c|c|c|c|c|c|c|c|c|}
\hline PROM & References & $\begin{array}{l}\text { Construct } \\
\text { validity } \\
\text { (CFI) }\end{array}$ & $\begin{array}{l}\text { Internal } \\
\text { consistency } \\
\text { (Cronbach's } \\
\text { alpha) }\end{array}$ & $\begin{array}{l}\text { Reliability } \\
\text { (ICC) }\end{array}$ & $\begin{array}{l}\text { Measurement } \\
\text { error }\end{array}$ & $\begin{array}{l}\text { Hypothesis } \\
\text { testing for } \\
\text { construct } \\
\text { validity }\end{array}$ & $\begin{array}{l}\text { Cross-cultural } \\
\text { validity } \\
\text { measurement } \\
\text { invariance }\end{array}$ & $\begin{array}{l}\text { Criterion } \\
\text { validity }\end{array}$ & Responsiveness \\
\hline \multirow{5}{*}{$\begin{array}{l}\text { MOS-HIV } \\
\text { Chinese } \\
\text { version }\end{array}$} & $\begin{array}{l}\text { Lau et al. } \\
\text { [36] }\end{array}$ & - & $+(0.78-0.90)$ & $\begin{array}{l}+(0.50- \\
0.84)\end{array}$ & $N R$ & $N R$ & $N R$ & $N R$ & $N R$ \\
\hline & $\begin{array}{l}\text { Liu et al. } \\
\text { [37] }\end{array}$ & $+(0.97)$ & $+(0.79-0.93)$ & $\begin{array}{l}+(0.87- \\
0.89)\end{array}$ & $N R$ & + & $N R$ & $N R$ & $N R$ \\
\hline & $\begin{array}{l}\text { Dong et al. } \\
\text { [38] }\end{array}$ & - & $+(0.81)$ & $N R$ & $N R$ & $N R$ & $N R$ & $N R$ & $N R$ \\
\hline & $\begin{array}{l}\text { Yang et al. } \\
\text { [39] }\end{array}$ & - & $+(0.67-0.86)$ & $N R$ & $N R$ & $N R$ & NR & $N R$ & NR \\
\hline & Yu et al. [40] & - & $+(0.69-0.87)$ & $\begin{array}{l}+(0.73- \\
0.88)\end{array}$ & $N R$ & + & $N R$ & NR & $N R$ \\
\hline $\begin{array}{l}\text { MOS-HIV } \\
\text { Lugandan } \\
\text { version }\end{array}$ & $\begin{array}{l}\text { Mast et al. } \\
\text { [41] }\end{array}$ & - & $+(0.51-0.84)$ & NR & $N R$ & $N R$ & + & NR & $N R$ \\
\hline $\begin{array}{l}\text { MOS-HIV } \\
\text { Italian ver- } \\
\text { sion }\end{array}$ & $\begin{array}{l}\text { Schifano } \\
\text { et al. [44] }\end{array}$ & - & $+(>0.80)$ & $N R$ & NR & $?$ & NR & $N R$ & NR \\
\hline $\begin{array}{l}\text { MOS-HIV } \\
\text { Korean ver- } \\
\text { sion }\end{array}$ & $\begin{array}{l}\text { Shim et al. } \\
\text { [45] }\end{array}$ & $+(0.97)$ & $+(0.78-0.95)$ & $N R$ & NR & + & + & $N R$ & $N R$ \\
\hline $\begin{array}{l}\text { MOS-HIV } \\
\text { Ugandan } \\
\text { version }\end{array}$ & $\begin{array}{l}\text { Stangl et al. } \\
{[46]}\end{array}$ & - & $+(0.79-0.91)$ & $N R$ & $N R$ & $?$ & + & NR & - \\
\hline $\begin{array}{l}\text { MOS-HIV } \\
\text { Shona ver- } \\
\text { sion }\end{array}$ & $\begin{array}{l}\text { Taylor et al. } \\
{[47]}\end{array}$ & - & $+(0.60-0.86)$ & $N R$ & $N R$ & + & NR & $N R$ & $N R$ \\
\hline \multirow[t]{2}{*}{ MQoL-HIV } & $\begin{array}{l}\text { Remple } \\
\text { et al. [66] }\end{array}$ & $N R$ & $+(0.43-0.92)$ & $\begin{array}{l}+(0.60- \\
0.96)\end{array}$ & $N R$ & + & NR & $N R$ & NR \\
\hline & $\begin{array}{l}\text { Smith et al. } \\
\text { [67] }\end{array}$ & - & $+(0.56-0.86)$ & $\begin{array}{l}+(0.64- \\
0.88)\end{array}$ & $N R$ & $N R$ & $N R$ & $N R$ & + \\
\hline $\begin{array}{l}\text { MQoL-HIV } \\
\text { German } \\
\text { version }\end{array}$ & $\begin{array}{l}\text { Kemmler } \\
\text { et al. [10] }\end{array}$ & - & $+(0.61-0.85)$ & $\begin{array}{l}+(0.74- \\
0.89)\end{array}$ & $N R$ & + & NR & $N R$ & NR \\
\hline $\begin{array}{l}\text { MQoL-HIV } \\
\text { Japanese } \\
\text { version }\end{array}$ & $\begin{array}{l}\text { Watanabe } \\
\text { et al. [68] }\end{array}$ & - & $+(0.47-0.85)$ & $N R$ & NR & NR & NR & - & NR \\
\hline FAHI & $\begin{array}{l}\text { Peterman } \\
\text { et al. [15] }\end{array}$ & - & $+(0.91)$ & $N R$ & NR & $?$ & + & $N R$ & $N R$ \\
\hline \multirow[t]{2}{*}{ HAT-QoL } & $\begin{array}{l}\text { Holmes and } \\
\text { Shea et al. } \\
{[76]}\end{array}$ & - & $+(>0.80)$ & $N R$ & $N R$ & $?$ & NR & NR & NR \\
\hline & $\begin{array}{l}\text { Holmes and } \\
\text { Shea et al. } \\
{[16]}\end{array}$ & - & $+(0.80-0.89)$ & $N R$ & $N R$ & $N R$ & $N R$ & $N R$ & NR \\
\hline $\begin{array}{l}\text { HAT-QoL } \\
\text { Shona ver- } \\
\text { sion }\end{array}$ & $\begin{array}{l}\text { Taylor et al. } \\
{[47]}\end{array}$ & - & $+(0.63-0.85)$ & $N R$ & $N R$ & $?$ & $N R$ & $N R$ & $N R$ \\
\hline $\begin{array}{l}\text { HIV/ } \\
\text { AIDSQoL } \\
\text { Chinese } \\
\text { version }\end{array}$ & $\begin{array}{l}\text { Zhang et al. } \\
{[17]}\end{array}$ & - & $+(0.94)$ & $+(0.80)$ & NR & NR & NR & NR & + \\
\hline $\begin{array}{l}\text { HIV-QL31 } \\
\text { French ver- } \\
\text { sion }\end{array}$ & $\begin{array}{l}\text { Leplège } \\
\text { et al. [11] }\end{array}$ & - & $+(0.93)$ & NR & NR & NR & NR & $N R$ & NR \\
\hline
\end{tabular}


Table 4 (continued)

\begin{tabular}{|c|c|c|c|c|c|c|c|c|c|}
\hline PROM & References & $\begin{array}{l}\text { Construct } \\
\text { validity } \\
\text { (CFI) }\end{array}$ & $\begin{array}{l}\text { Internal } \\
\text { consistency } \\
\text { (Cronbach's } \\
\text { alpha) }\end{array}$ & $\begin{array}{l}\text { Reliability } \\
\text { (ICC) }\end{array}$ & $\begin{array}{l}\text { Measurement } \\
\text { error }\end{array}$ & $\begin{array}{l}\text { Hypothesis } \\
\text { testing for } \\
\text { construct } \\
\text { validity }\end{array}$ & $\begin{array}{l}\text { Cross-cultural } \\
\text { validity } \\
\text { measurement } \\
\text { invariance }\end{array}$ & $\begin{array}{l}\text { Criterion } \\
\text { validity }\end{array}$ & Responsiveness \\
\hline $\begin{array}{l}\text { HOPES } \\
\text { Dutch, Eng- } \\
\text { lish version }\end{array}$ & $\begin{array}{l}\text { De Boer } \\
\text { et al. [14] }\end{array}$ & - & $+(0.80-0.92)$ & $N R$ & NR & $?$ & NR & - & $N R$ \\
\hline $\begin{array}{l}\text { PROQOL- } \\
\text { HIV }\end{array}$ & $\begin{array}{l}\text { Herrmann } \\
\text { et al. [71] }\end{array}$ & $N R$ & $+(0.94)$ & $+(0.86)$ & $N R$ & $N R$ & $N R$ & NR & $N R$ \\
\hline $\begin{array}{l}\text { PROQOL- } \\
\text { HIV English, } \\
\text { Brazilian, } \\
\text { Cambodian, } \\
\text { Chinese, } \\
\text { French, } \\
\text { Senegalese, } \\
\text { and Thai } \\
\text { versions }\end{array}$ & $\begin{array}{l}\text { Duracinsky } \\
\text { et al. [12] }\end{array}$ & - & $+(0.77-0.89)$ & $+(0.86)$ & $N R$ & $?$ & $N R$ & $N R$ & $N R$ \\
\hline $\begin{array}{l}\text { HIV QoL } \\
\text { Scale-1 }\end{array}$ & $\begin{array}{l}\text { Xiang et al. } \\
\text { [77-79] }\end{array}$ & - & $+(0.65-0.7)$ & $+(>0.7)$ & $N R$ & $N R$ & $N R$ & $N R$ & $N R$ \\
\hline $\begin{array}{l}\text { HIV QoL } \\
\text { Scale-2 }\end{array}$ & $\begin{array}{l}\text { Meng et al. } \\
{[80]}\end{array}$ & NR & $+(0.90)$ & $+(0.80)$ & $N R$ & $N R$ & $N R$ & - & $N R$ \\
\hline $\begin{array}{l}\text { HIV QoL } \\
\text { Scale-3 }\end{array}$ & Su et al. [81] & - & $+(0.94)$ & $+(0.80)$ & NR & $N R$ & NR & NR & NR \\
\hline $\begin{array}{l}\text { HIV QoL } \\
\text { Scale-4 }\end{array}$ & $\begin{array}{l}\text { Guo et al. } \\
\text { [82] }\end{array}$ & - & $+(0.94)$ & $+(0.97)$ & $N R$ & NR & $N R$ & NR & $N R$ \\
\hline \multirow{2}{*}{$\begin{array}{l}\text { WHOQOL } \\
\text { Chinese } \\
\text { version }\end{array}$} & $\begin{array}{l}\text { Fang et al. } \\
\text { [22] }\end{array}$ & - & $+(0.74-0.85)$ & $\begin{array}{l}+(0.51- \\
0.78)\end{array}$ & $N R$ & $?$ & $N R$ & NR & $N R$ \\
\hline & $\begin{array}{l}\text { Liu et al. } \\
\text { [62] }\end{array}$ & - & - & NR & NR & $N R$ & NR & NR & NR \\
\hline $\begin{array}{l}\text { WHOQOL } \\
\text { Malay ver- } \\
\text { sion }\end{array}$ & $\begin{array}{l}\text { Saddkia } \\
\text { et al. [9] }\end{array}$ & - & $+(0.93)$ & $+(0.87)$ & NR & + & + & NR & NR \\
\hline $\begin{array}{l}\text { WHOQOL- } \\
\text { BREF } \\
\text { Nigerian } \\
\text { version }\end{array}$ & $\begin{array}{l}\text { Akinboro } \\
\text { et al. [63] }\end{array}$ & NR & $+(0.85)$ & $N R$ & $N R$ & $N R$ & $N R$ & NR & $N R$ \\
\hline $\begin{array}{l}\text { WHOQOL- } \\
\text { BREF }\end{array}$ & $\begin{array}{l}\text { Thompson } \\
\text { et al. [64] }\end{array}$ & $+(0.89)$ & $+(0.65-0.78)$ & $N R$ & $N R$ & + & $N R$ & NR & $N R$ \\
\hline $\begin{array}{l}\text { MOS Indic } \\
\text { version }\end{array}$ & $\begin{array}{l}\text { Kohli et al. } \\
\text { [23] }\end{array}$ & - & $+(>0.75)$ & $N R$ & $N R$ & $N R$ & $N R$ & $N R$ & $N R$ \\
\hline MOS SF-20 & $\begin{array}{l}\text { Smith et al. } \\
{[65]}\end{array}$ & - & $+(0.76-0.89)$ & $N R$ & $N R$ & $N R$ & + & $N R$ & + \\
\hline $\begin{array}{l}\text { MVQoLI } \\
\text { Uganda } \\
\text { version }\end{array}$ & $\begin{array}{l}\text { Namisango } \\
\text { et al. [24] }\end{array}$ & NR & $+(0.85)$ & $N R$ & $N R$ & + & + & $N R$ & $N R$ \\
\hline EQ-5D & $\begin{array}{l}\text { Wu et al. } \\
{[21]}\end{array}$ & - & - & $N R$ & $N R$ & $?$ & NR & NR & + \\
\hline $\begin{array}{l}\text { EQ-5D-5L } \\
\text { Vietnamese } \\
\text { version }\end{array}$ & $\begin{array}{l}\text { Tran et al. } \\
{[20]}\end{array}$ & - & $+(0.85)$ & $N R$ & $N R$ & $?$ & NR & NR & NR \\
\hline $\begin{array}{l}\text { EUROHIS- } \\
\text { QoL-8 } \\
\text { Portuguese } \\
\text { version }\end{array}$ & $\begin{array}{l}\text { Pereira and } \\
\text { Canavarro } \\
{[75]}\end{array}$ & $+(0.89)$ & $+(0.85)$ & $N R$ & $N R$ & + & $N R$ & NR & NR \\
\hline HAQ-DI & $\begin{array}{l}\text { Sousa et al. } \\
{[26]}\end{array}$ & $+(0.974)$ & $N R$ & $N R$ & NR & $N R$ & $N R$ & NR & NR \\
\hline HUI3 & $\begin{array}{l}\text { Nosyk et al. } \\
{[28]}\end{array}$ & NR & $N R$ & $N R$ & $N R$ & + & $N R$ & - & + \\
\hline
\end{tabular}


Table 4 (continued)

\begin{tabular}{|c|c|c|c|c|c|c|c|c|c|}
\hline PROM & References & $\begin{array}{l}\text { Construct } \\
\text { validity } \\
\text { (CFI) }\end{array}$ & $\begin{array}{l}\text { Internal } \\
\text { consistency } \\
\text { (Cronbach's } \\
\text { alpha) }\end{array}$ & $\begin{array}{l}\text { Reliability } \\
\text { (ICC) }\end{array}$ & $\begin{array}{l}\text { Measurement } \\
\text { error }\end{array}$ & $\begin{array}{l}\text { Hypothesis } \\
\text { testing for } \\
\text { construct } \\
\text { validity }\end{array}$ & $\begin{array}{l}\text { Cross-cultural } \\
\text { validity } \\
\text { measurement } \\
\text { invariance }\end{array}$ & $\begin{array}{l}\text { Criterion } \\
\text { validity }\end{array}$ & Responsiveness \\
\hline $\begin{array}{l}\text { ISSQoL Ital- } \\
\text { ian version }\end{array}$ & $\begin{array}{l}\text { Bucciardini } \\
\text { et al. [69] }\end{array}$ & - & $+(>0.70)$ & NR & $N R$ & $N R$ & $N R$ & NR & $N R$ \\
\hline PozQoL & $\begin{array}{l}\text { Brown et al. } \\
{[70]}\end{array}$ & $+(>0.95)$ & $+(0.95)$ & $+(0.95)$ & NR & + & $N R$ & NR & $N R$ \\
\hline PROMIS-29 & $\begin{array}{l}\text { Schnall } \\
\text { et al. [25] }\end{array}$ & - & $+(0.87-0.97)$ & $\begin{array}{l}+(0.61- \\
0.81)\end{array}$ & $N R$ & $N R$ & $N R$ & NR & NR \\
\hline QWB scale & $\begin{array}{l}\text { Kaplan et al. } \\
\text { [27] }\end{array}$ & NR & NR & NR & NR & $N R$ & $N R$ & - & $N R$ \\
\hline $\begin{array}{l}\text { SF-36v2 } \\
\text { Brazilian- } \\
\text { Portuguese } \\
\text { version }\end{array}$ & $\begin{array}{l}\text { Kusterer } \\
\text { et al. [19] }\end{array}$ & $+(0.95)$ & NR & NR & NR & NR & NR & NR & NR \\
\hline \multirow[t]{2}{*}{ SF-36 } & $\begin{array}{l}\text { Riley et al. } \\
\text { [72] }\end{array}$ & - & $+(0.77-0.90)$ & $N R$ & NR & $?$ & $N R$ & NR & $N R$ \\
\hline & $\begin{array}{l}\text { Turner- } \\
\text { Bowker } \\
\text { et al. [73] }\end{array}$ & NR & $N R$ & $N R$ & $N R$ & $?$ & $N R$ & $N R$ & $N R$ \\
\hline $\begin{array}{l}\text { SF-36 Chi- } \\
\text { nese version }\end{array}$ & $\begin{array}{l}\text { Zhang et al. } \\
\text { [74] }\end{array}$ & - & $+(0.928)$ & $N R$ & $N R$ & + & $N R$ & NR & $N R$ \\
\hline $\begin{array}{l}\text { SF-12 } \\
\text { Kiswahili } \\
\text { version }\end{array}$ & $\begin{array}{l}\text { Patel et al. } \\
\text { [18] }\end{array}$ & - & $N R$ & $N R$ & $N R$ & $?$ & $N R$ & - & $N R$ \\
\hline
\end{tabular}

"+", sufficient; "-", insufficient; "?", indeterminate; CFI, Comparative fit index; EQ-5D, EuroQol-5 Dimensions; EUROHIS-QoL-8, European health interview surveysquality of life-8; FAHI, Functional Assessment of HIV Infection; HAT-QoL, HIV/AIDS Targeted Quality of Life; HAQ-DI, Health Assessment Questionnaire Disability Index; HIV-OL31, HIV Disease Quality of Life 31-Item Instrument; HIV/AIDSQoL, HIV/AIDS Quality of Life Questionnaire; HOPES, HIV Overview of Problems Evaluation Scale; HUI3, Health Utility Index 3; ICC, Intra-class correlation coefficients; ISSQoL, The Italian National Institute of Health Quality of Life; MQoL-HIV, Multidimensional Quality of Life Questionnaire for Persons with HIV/AIDS; MOS, Medical Outcomes Study; MOS-HIV, Medical Outcomes Study HIV Health Survey; MVQoLI, Missoula-Vitas Quality-of-Life Index; NR, not reported; PLWH, people living with HIV; PROM , Patient-reported outcome measure; PROMIS Patient-Reported Outcomes Measurement Information System; PROQoL-HIV, Patient-Reported Outcomes Quality of Life-HIV instrument; QWB, Quality of Well-Being scale; SF, Short Form Health Survey; WHOQoL, World Health Organization's Quality of Life; WHOQoL-BREF, The brief version of the World Health Organization's Quality of Life. WHOQoL-HIV, World Health Organization's Quality of Life Instrument in HIV Infection; WHOQoL-HIV-BREF, Thebrief version of the World Health Organization's Quality of Life Instrument in HIV Infection

validity and discriminant validity were reported in several reviews [31, 32]. As one of the earliest HIV-specific HRQoL PROMs, MOS-HIV has been translated into at least 14 languages. The reliability and validity of the instrument were likely to decrease in the different translated versions due to their cultural adjustment. For these versions, mixed findings on the hypothesis testing of the MOS-HIV were reported [34-47]. As data on the psychometric properties of many studies were missing or indeterminate, we can draw only preliminary conclusions. More research is needed to fill the gap in the research on the psychometric properties of the existing instruments on HRQoL in PLWH.

Our review found that, in addition to MOS-HIV, the WHOQoL-HIV-BREF was reported to have good psychometric properties. Seven of eight different language versions of the WHOQoL-HIV-BREF were rated as "high" in hypothesis testing for construct validity. The WHOQoL-HIV-BREF was reported to have better reliability and internal consistency than other instruments except the MOS-HIV. Two language versions of the WHOQoL-HIV-BREF were rated as "very low" in internal consistency. Three language versions were rated as "very low", and two were rated as "moderate" in construct validity. Connell and Skevington published a study to report the development and psychometric properties of the WHOQoL-HIV-BREF [51]. The results showed very good discriminant validity, which suggested the important role of the WHOQoL-HIV-BREF in distinguishing different stages of HIV disease progression [51].

Although the MOS-HIV showed good psychometric properties, a major advantage of the WHOQoL-HIVBREF is its brevity. It contains only 31 items, and most participants can complete the instrument in $8 \mathrm{~min}$. The WHOQoL-HIV-BREF is increasingly being used in HIV research. From a practical perspective, the MOS-HIV and WHOQoL-HIV-BREF focus on different dimensions and are based on different theoretical perspectives. The MOS-HIV is a multidimensional assessment measure that assesses physical, psychological, and social 


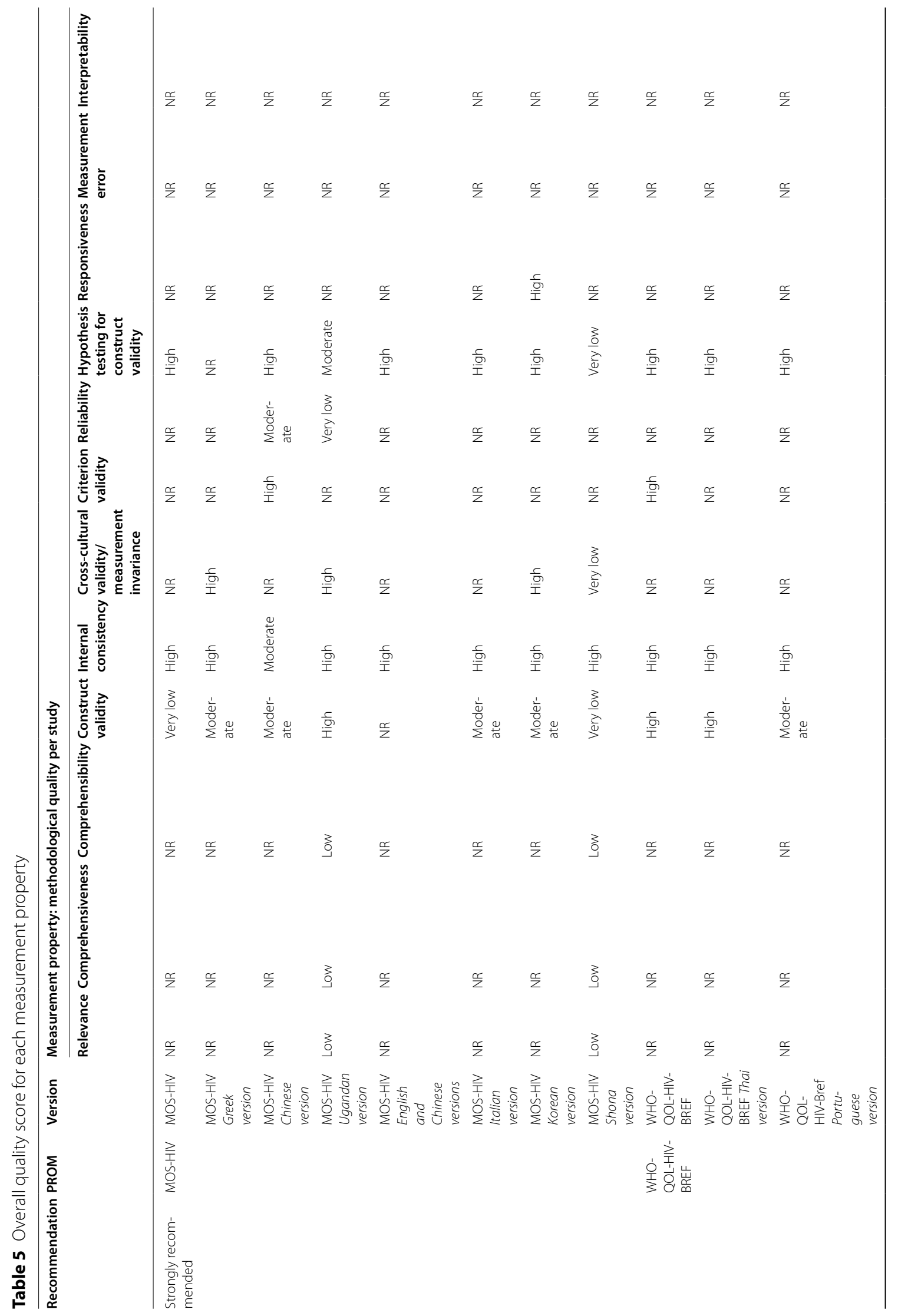




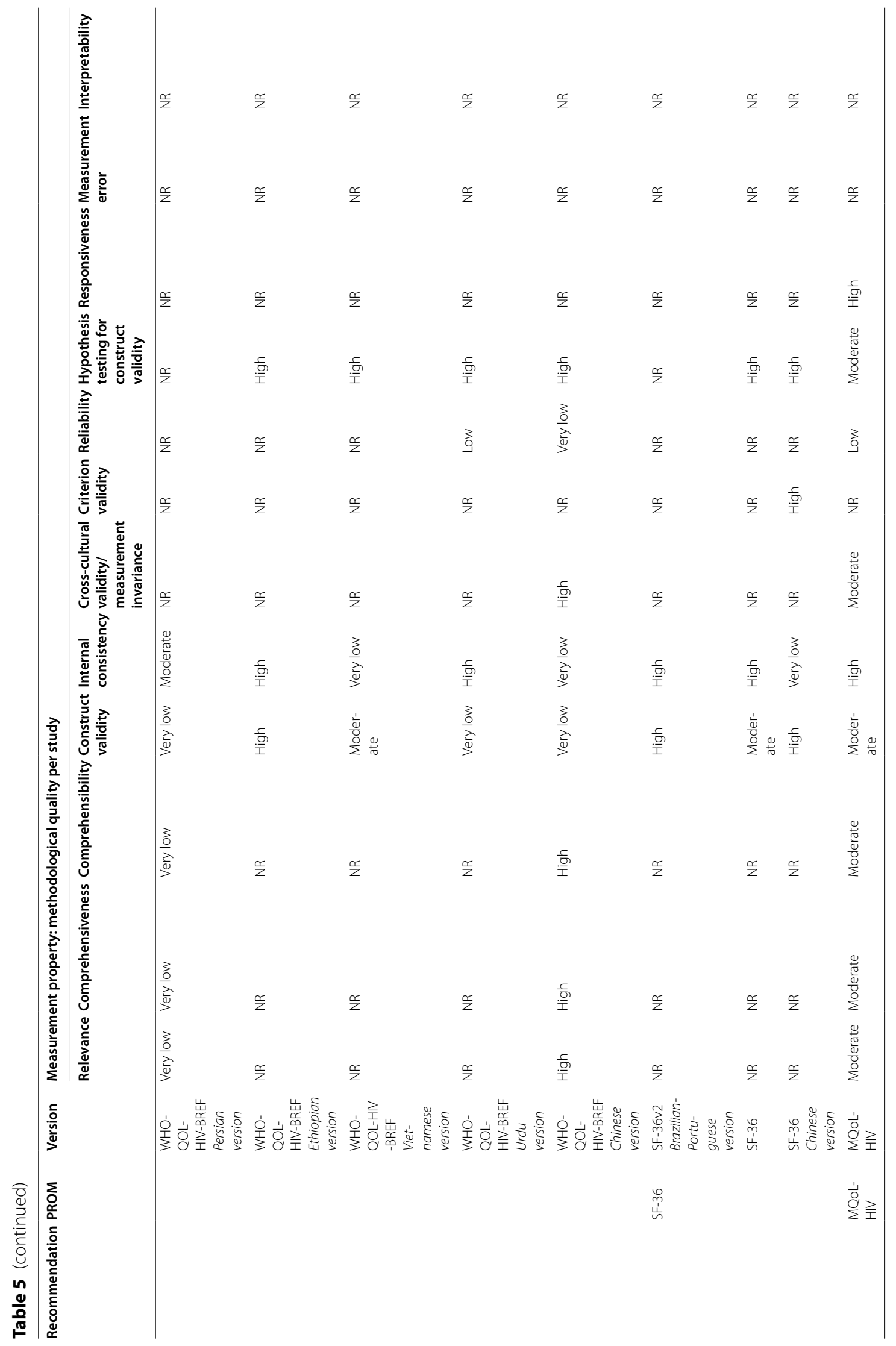




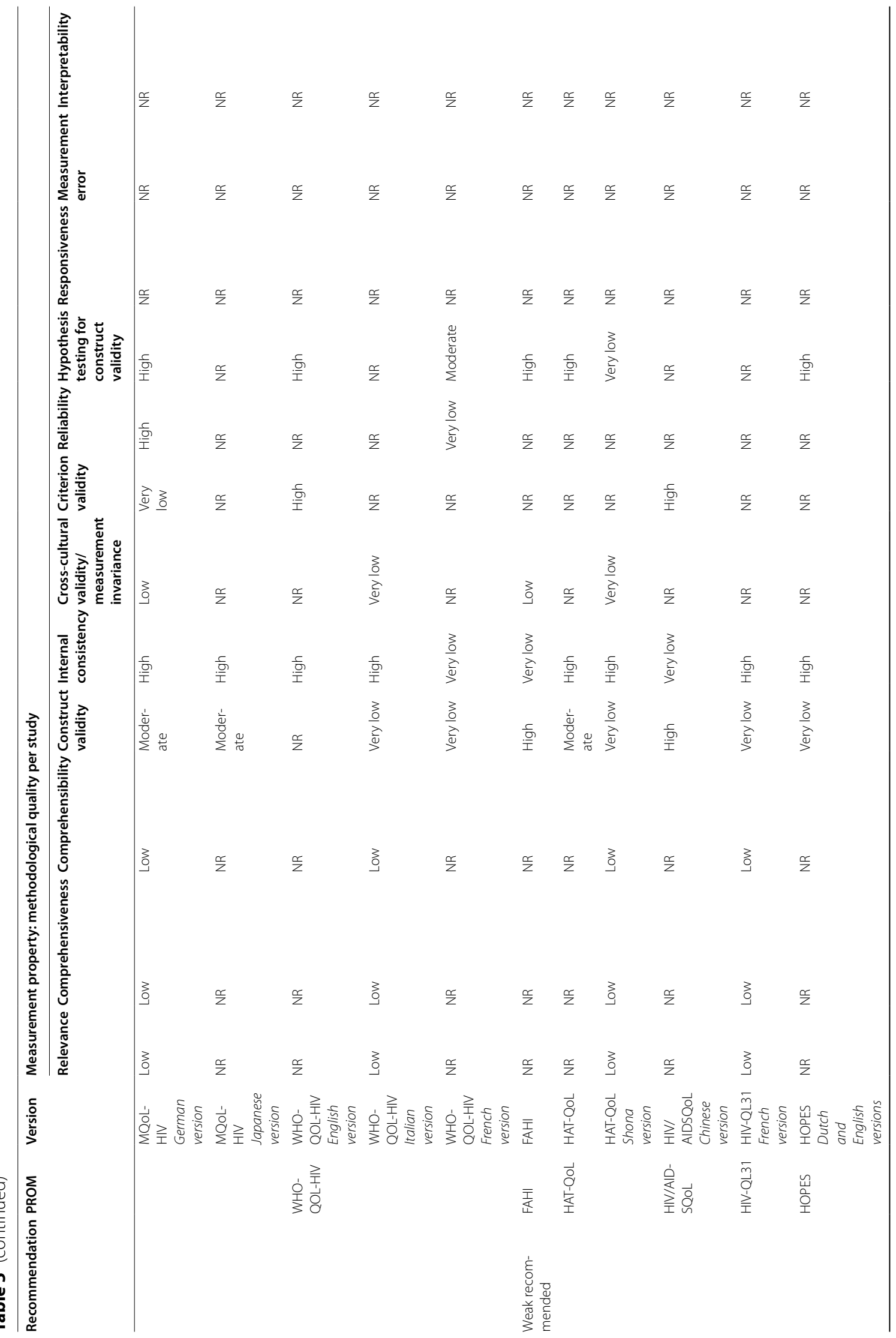




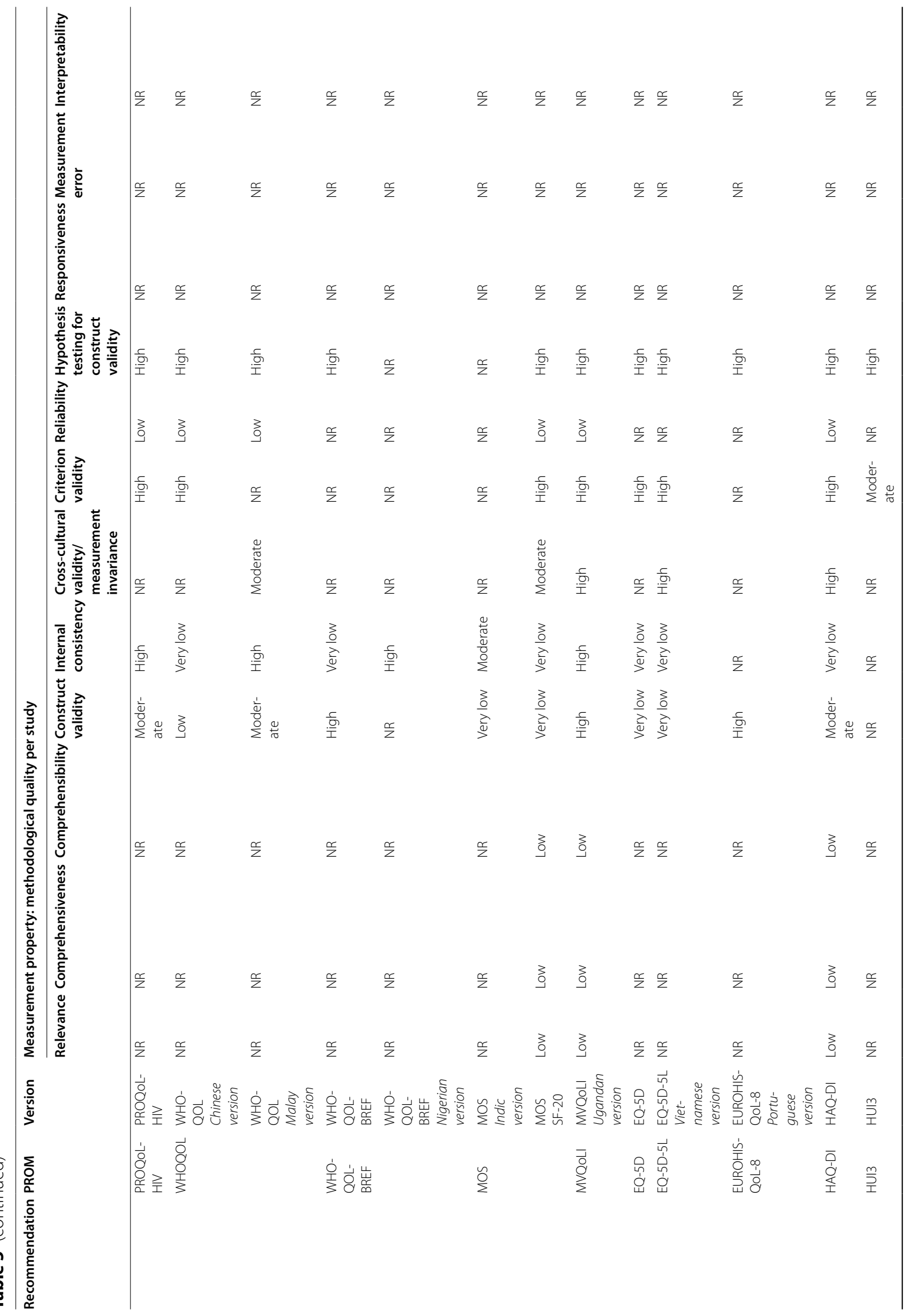




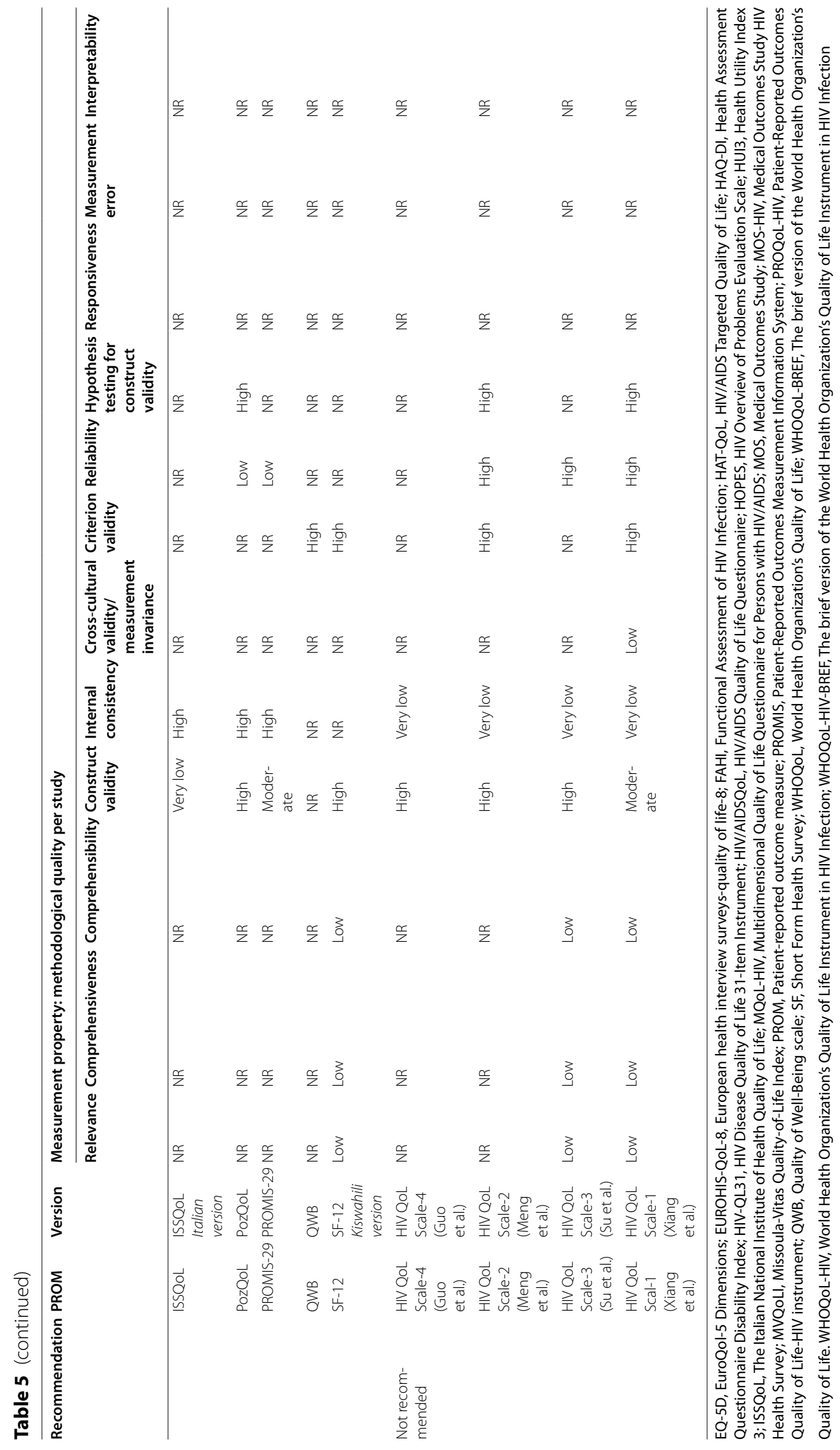


functioning. The MOS-HIV consists of 35 items across 11 domains: physical functioning, pain, social functioning, role functioning, emotional well-being, energy/ fatigue, cognitive function, health distress, health transition, general health, and overall quality of life [8]. The WHOQoL-HIV-BREF has 31 items across six domains: physical functioning, psychological functioning, levels of independence, social relationships, environment, and spirituality [9].

The SF-36 is an internationally used generic instrument that can provide a comprehensive assessment of HRQoL in various populations. Although the SF-36 is also widely used in PLWH, only four validation studies were found in PLWH [19, 72-74]. The number of validation studies of different language versions was fewer than that of WHOQoL-HIV-BREF and MOS-HIV. From a global perspective, a better PROM should report decent psychometric properties in all language versions. Future studies are warranted to conduct validation studies evaluating the psychometric properties of the SF-36 in PLWH in various contexts. In addition, other aspects, such as scoring methods and content of items, may also restrict the wide usage of PLWH [85, 86]. Skevington et al. concluded that the SF-36 includes several different scoring scales and response options, which may complicate scoring and thus limit the widespread clinical use of the SF-36 [85]. Abbasi-Ghahramanloo et al. showed that the SF-36 may lack the ability to measure self-reported subjective HRQoL [86].

This study strongly recommends four HIV-specific and one generic PROM. Generic PROMs can be used to measure the HRQoL of general or HIV-infected populations. However, they may lack the sensitivity to detect subtle changes specific to PLWH, including stigma, relationship issues, and comorbidities [87]. HIV-specific PROMs are more closely related to the disease than generic PROMs and have the sensitivity and specificity needed for HIV-specific domains. Nonetheless, they are not conducive to use in comparisons across populations $[88,89]$. It is highly recommended that when selecting instruments, researchers need to consider more aspects, including psychometric properties, instrument content coverage, ease of use, and scoring methods. Therefore, the choice of PROMs should be based on the specific aims of assessments and the response burden for participants.

Overall, we acknowledge that there are some limitations to this study. First, this study included only articles published in English or Chinese. Therefore, some studies published in other languages may not have been included, which may have affected the conclusions of this review. Second, we included only studies that aimed to evaluate the measurement properties of PROMs in PLWH. Some cross-sectional studies that aimed to explore the level of HRQoL in PLWH also reported the reliability and validity of PROMs. These types of studies were not included in this study. Third, we included four PROMs in Chinese that did not report a specific name. We used "unknown" to describe the names of these PROMs in all tables.

\section{Conclusions}

This systematic review identified and described the psychometric properties of 30 instruments and 69 studies. The findings from the included studies highlighted that compared to other HIV-specific and generic HRQoL PROMs, the MOS-HIV had the best psychometric properties and could be recommended as the most suitable for use in research and clinics. We also strongly recommended using WHOQoL-HIV-BREF, SF-36, MQoL-HIV, and WHOQoL-HIV to evaluate HRQoL in PLWH. We suggest that the choice of PROMs should be based on the specific aims of assessments and the response burden for participants.

\section{Abbreviations}

ART: Antiretroviral therapy; COSMIN: Consensus-based standards for the selection of health measurement instruments; EQ-5D: EuroQol-5 dimensions; EUROHIS-QoL-8: European health interview surveys-quality of life-8; FAHI: Functional assessment of HIV infection; HAT-QoL: HIV/AIDS targeted quality of life; GRADE: Grading, recommendations, assessment, development, and evaluation; HAQ-DI: Health Assessment Questionnaire Disability Index; HIV-QL31: HIV disease quality of life 31-item instrument; HIV/AIDSQoL: HIV/ AIDS quality of life questionnaire; HOPES: HIV Overview of Problems Evaluation Scale; HRQoL: Health-related quality of life; HUI3: Health Utility Index 3; ISSQOL: The Italian National Institute of Health Quality of Life; MQOL-HIV: Multidimensional quality of life questionnaire for persons with HIV/AIDS; MOS: Medical outcomes study; MOS-HIV: Medical outcomes study HIV health survey; MVQoLI: Missoula-Vitas Quality-of-Life Index; PLWH: People living with HIV; PROM: Patient-reported outcome measure; PROMIS: Patient-reported outcomes measurement information system; PROQoL-HIV: Patient-reported outcomes quality of life-HIV instrument; QWB: Quality of well-being scale; SF: Short form health survey; WHOQoL: World Health Organization's Quality of Life; WHOQoL-BREF: The brief version of the World Health Organization's Quality of Life; WHOQoL-HIV: World Health Organization's Quality of Life Instrument in HIV Infection; WHOQoL-HIV-BREF: The brief version of the World Health Organization's Quality of Life Instrument in HIV Infection.

\section{Supplementary Information}

The online version contains supplementary material available at https://doi. org/10.1186/s12955-021-01910-w.

Additional file 1. Searching Strategies and Results.

Additional file 2. PRISMA 2020 Checklist.

\section{Acknowledgements}

Not applicable.

\section{Authors' contributions}

Performed the search, provided initial tables, and wrote the original draft version. HW; critically evaluated the manuscript and tables, project administration, ZZ; supervised the literature search, helped in writing, ZY; writingreviewing and editing, $\mathrm{SH}, \mathrm{YH}, \mathrm{LZ}$. All authors read and approved the final manuscript. 


\section{Funding}

This work is supported by the Ministry of Education of Humanities and Social Science Project (20YJCZH254), Shanghai Soft Science Research Program (72104051), Shanghai Sailing Program (20YF1401800), China Medical Board Open Competition Program (\#20-372), and National Natural Science Foundation of China (72104051). The funders had no involvement in or influence on this systematic review.

\section{Availability of data and materials Not applicable.}

\section{Declarations}

\section{Ethics approval and consent to participate}

Not applicable.

\section{Consent for publication}

All participants provided written informed consent to publication.

\section{Competing interests}

The authors declare that they have no competing interests.

\section{Author details}

${ }^{1}$ Fudan University School of Public Health, Shanghai, China. ${ }^{2}$ School of Nursing, Fudan University, Shanghai, China. ${ }^{3}$ Fudan University Centre for EvidenceBased Nursing: A Joanna Briggs Institute Centre of Excellence, 305 Fenglin Rd, Shanghai 200032, China. ${ }^{4}$ Peking University School of Nursing, Beijing, China.

${ }^{5}$ Shanghai Public Health Clinical Center, Shanghai, China.

Received: 7 July 2021 Accepted: 14 December 2021

Published online: 10 January 2022

\section{References}

1. Hays RD, Reeve BB. Measurement and modeling of health-related quality of life. In: Killewo J, Heggenhougen HK, Quah SR, editors. International Encyclopedia of public health. 2nd ed. San Diego: Academic Press; 2017.

2. Torrance GW. Utility approach to measuring health-related quality of life. J Chronic Dis. 1987:40(6):593-600.

3. Jang HJ, Satre DD, Leyden W, Leibowitz A, Silverberg MJ. Mental and physical quality of life by age groups in people living with HIV. J Assoc Nurses AIDS Care. 2019;30(5):500-10.

4. Lazarus JV, Safreed-Harmon K, Barton SE, Costagliola D, Dedes N, del Amo $\mathrm{VJ}$, et al. Beyond viral suppression of HIV-the new quality of life frontier. BMC Med. 2016;14(1):94-8.

5. Webster P. UNAIDS survey aligns with so-called fourth 90 for HIV/AIDS. Lancet. 2019;393(10187):2188.

6. The Lancet HIV. Living well with HIV. Lancet HIV. 2019;6(12):e807.

7. Kall M, Marcellin F, Harding R, Lazarus JV, Carrieri P. Patient-reported outcomes to enhance person-centred HIV care. Lancet HIV. 2019;7(1):e59-68.

8. Wu AW, Revicki DA, Jacobson D, Malitz FE. Evidence for reliability, validity and usefulness of the medical outcomes study HIV health survey (MOSHIV). Qual Life Res. 1997;6(6):481-93.

9. Saddki N, Noor MM, Norbanee TH, et al. Validity and reliability of the Malay version of WHOQOL-HIV BREF in patients with HIV infection. AIDS Care. 2009:21(10):1271-8.

10. Kemmler G, Schmied B, Shetty-Lee A, et al. Quality of life of HIV-infected patients: psychometric properties and validation of the German version of the MQOL-HIV. Qual Life Res. 2003;12(8):1037-50.

11. Leplège $A$, Rude N, Ecosse E, Ceinos R, Dohin E, Pouchot J. Measuring quality of life from the point of view of HIV-positive subjects: the HIVQL31. Qual Life Res. 1997;6(6):585-94.

12. Duracinsky M, Lalanne C, Le Coeur S, et al. Psychometric validation of the PROQOL-HIV questionnaire, a new health-related quality of life instrument-specific to HIV disease. J Acquir Immune Defic Syndr. 2012;59(5):506-15.

13. O'Connell K, Skevington S, Saxena S, WHOQOL HIV Group. Preliminary development of the World Health Organsiation's Quality of Life HIV instrument (WHOQOL-HIV): analysis of the pilot version. Soc Sci Med. 2003;57(7):1259-75.

14. De Boer JB, Sprangers MA, Aaronson NK, Lange JM, van Dam FS. A study of the reliability, validity and responsiveness of the HIV overview of problems evaluation system (HOPES) in assessing the quality of life of patients with AIDS and symptomatic HIV infection. Qual Life Res. 1996;5(3):339-47.

15. Peterman AH, Cella D, Mo F, McCain N. Psychometric validation of the revised Functional Assessment of Human Immunodeficiency Virus Infection (FAHI) quality of life instrument. Qual Life Res. 1997:6(6):572-84.

16. Holmes WC, Shea JA. Performance of a new, HIV/AIDS-targeted quality of life (HAT-QoL) instrument in asymptomatic seropositive individuals. Qual Life Res. 1997:6(6):561-71.

17. Zhang ML, Wei JY, Wu YM, et al. Evaluation of HIV/AIDS qol-46. Chin Med Res. 2009:22(011):10-3.

18. Patel AR, Lester RT, Marra CA, et al. The validity of the SF-12 and SF-6D instruments in people living with HIV/AIDS in Kenya. Health Qual Life Outcomes. 2017;15(1):143.

19. Lins-Kusterer L, Valdelamar J, Aguiar CVN, Menezes MS, Netto EM, Brites C. Validity and reliability of the 36-item short form health survey questionnaire version 2 among people living with HIV in Brazil. Braz J Infect Dis. 2019;23(5):313-21.

20. Tran BX, Ohinmaa A, Nguyen LT. Quality of life profile and psychometric properties of the EQ-5D-5L in HIV/AIDS patients. Health Qual Life Outcomes. 2012;10:132

21. Wu AW, Jacobson KL, Frick KD, et al. Validity and responsiveness of the euroqol as a measure of health-related quality of life in people enrolled in an AIDS clinical trial. Qual Life Res. 2002;11(3):273-82.

22. Fang CT, Hsiung PC, Yu CF, Chen MY, Wang JD. Validation of the World Health Organization quality of life instrument in patients with HIV infection. Qual Life Res. 2002;11(8):753-62.

23. Kohli RM, Sane S, Kumar K, Paranjape RS, Mehendale SM. Modification of medical outcome study (MOS) instrument for quality of life assessment \& its validation in HIV infected individuals in India. Indian J Med Res. 2005;122(4):297-304.

24. Namisango E, Katabira E, Karamagi C, Baguma P. Validation of the Missoula-Vitas Quality-of-Life Index among patients with advanced AIDS in urban Kampala, Uganda. J Pain Symptom Manag. 2007;33(2):189-202.

25. Schnall R, Liu J, Cho H, Hirshfield S, Siegel K, Olender S. A Health-related quality-of-life measure for use in patients with HIV: a validation study. AIDS Patient Care STDs. 2017;31:43-8.

26. Sousa KH, Kwok OM, Ryu E, Cook SW. Confirmation of the validity of the HAQ-DI in two populations living with chronic illnesses. J Nurs Meas. 2008;16(1):31-42

27. Kaplan RM, Patterson TL, Kerner DN, Atkinson JH, Heaton RK, Grant I. The quality of well-being scale in asymptomatic HIV-infected patients. HNRC Group. HIV Neural Behavioral Research Center. Qual Life Res. 1997;6(6):507-14

28. Nosyk B, Sun H, Bansback N, et al. The concurrent validity and responsiveness of the health utilities index ( $\mathrm{HUI} 3$ ) among patients with advanced HIV/AIDS. Qual Life Res. 2009;18(7):815-24.

29. Cooper V, Clatworthy J, Harding R, Whetham J, Emerge Consortium. Measuring quality of life among people living with HIV: a systematic review of reviews. Health Qual Life Outcomes. 2017:15(1):220-39.

30. Clayson DJ, Wild DJ, Quarterman P, Duprat-Lomon I, Kubin M, Coons SJA. A comparative review of health-related quality-of-life measures for use in HIV/AIDS clinical trials. Pharmacoeconomics. 2006;24(8):751-65.

31. Gakhar H, Kamali A, Holodniy M. Health-related quality of life assessment after antiretroviral therapy: a review of the literature. Drugs. 2013;73(7):651-72.

32. Prinsen CAC, Vohra $S$, Rose MR, Al E. How to select outcome measurement instruments for outcomes included in a "core outcome set": a practical guideline. Trials. 2016;17(1):449-58.

33. Yang Z, Zhu Z, Wen H, et al. Psychometric properties of self-reported measures of health-related quality of life in people living with HIV: a systematic review protocol. JBI Evid Synth. 2021;19(10):2829-38.

34. Stasinopoulou PG, Tzavara C, Dimitrakaki C, et al. Reliability and validity of the Greek translation of the MOS-HIV health survey in HIV-infected individuals. Qual Life Res. 2010;19(2):199-205.

35. Hughes TE, Kaplan RM, Coons SJ, Draugalis JR, Johnson JA, Patterson $T L$. Construct validities of the quality of well-being scale and the 
MOS-HIV-34 Health Survey for HIV-infected patients. Med Decis Mak. 1997;17(4):439-46.

36. Lau JT, Tsui HY, Patrick LC, Rita CW, Molassiotis A. Validation of a Chinese version of the Medical Outcomes Study HIV Health Survey (MOS-HIV) among Chinese people living with HIV/AIDS in Hong Kong. Qual Life Res. 2006;15(6):1079-89.

37. Liu J, Zhu Y, Qu B. Reliability and validity of the Chinese version of the Medical Outcomes Study HIV Health Survey (MOS-HIV) in people living with HIV/AIDS (PLWHA) in China. PLoS ONE. 2018;13(7):e0201177.

38. Dong XJ, Lou LQ, Chen L, et al. Reliability and validity analysis of Chinese version of MOS-HIV scale. China Health Stat. 2015;32(4):619-620623

39. Yang F, Liu Q, Jia WD, et al. Reliability and validity of MOS-HIV scale. China Public Health. 2007:23(8):981-2.

40. Yu D, Liu M, Ming ZQ. Translation and revision of simplified Chinese version of MOS-HIV scale and evaluation of its reliability and validity. AIDS STD China. 2008;14(5):454-6.

41. Mast TC, Kigozi G, Wabwire-Mangen F, et al. Measuring quality of life among HIV-infected women using a culturally adapted questionnaire in Rakai district, Uganda. AIDS Care. 2004:16(1):81-94.

42. McDonnell KA, Gielen AC, Wu AW, O'Campo P, Faden R. Measuring health related quality of life among women living with HIV. Qual Life Res. 2000;9(8):931-40.

43. Paton NI, Chapman CA, Chan SP, et al. Validation of the Medical Outcomes Study HIV Health Survey as a measure of quality of life in HIV-infected patients in Singapore. Int J STD AIDS. 2002;13(7):456-61.

44. Schifano P, Borgia P, Wu AW, Spadea T, Milanese G, Perucci CA. Validity and reliability of the Italian translation of the MOS-HIV health survey in persons with AIDS. Qual Life Res. 2003;12(8):1137-46.

45. Shim EJ, Ha H, Lee SH, et al. Psychometric properties of the Korean version of the medical outcomes study HIV health survey: results from a multicenter survey in Korea. Health Qual Life Outcomes. 2018;16(1):92.

46. Stangl AL, Bunnell R, Wamai N, Masaba H, Mermin J. Measuring quality of life in rural Uganda: reliability and validity of summary scores from the medical outcomes study HIV health survey (MOS-HIV). Qual Life Res. 2012;21(9):1655-63.

47. Taylor TN, Dolezal C, Tross S, Holmes WC. Reliability and validity of two HIV/AIDS-specific quality of life instruments adapted for use in HIVpositive Zimbabweans. AIDS Care. 2009;21 (5):598-607.

48. Reychler G, Caty G, Vincent A, Billo S, Yombi JC. Validation of the French version of the World Health Organization quality of life HIV instrument. PLOS ONE. 2013;8(9):e73180.

49. Starace F, Cafaro L, Abrescia N, et al. Quality of life assessment in HIVpositive persons: application and validation of the WHOQOL-HIV, Italian version. AIDS Care. 2002;14(3):405-15.

50. Meemon N, Paek SC, Yenchai D, Wan TT. Application of the WHOQOL-HIV-BREF questionnaire in HIV-infected Thai patients: reliability and validity of the instrument. J Assoc Nurses AIDS Care. 2016;27(5):698-708.

51. O'Connell KA, Skevington SM. An international quality of life instrument to assess wellbeing in adults who are HIV-positive: a short form of the WHOQOL-HIV (31 items). AIDS Behav. 2012;16(2):452-60.

52. Pereira M, Martins A, Alves $S$, Canavarro MC. Assessing quality of life in middle-aged and older adults with HIV: psychometric testing of the WHOQOL-HIV-Bref. Qual Life Res. 2014;23(9):2473-9.

53. Salehi M, Niroumand S, Erfanian MR, Sajjadi RB, Dadgarmoghaddam M. Validation of Persian version of WHOQOL-HIV BREF questionnaire in Islamic Republic of Iran. East Mediterr Health J. 2016;22(9):647-53.

54. Tesfaye M, Olsen MF, Medhin G, Friis H, Hanlon C, Holm L. Adaptation and validation of the short version WHOQOL-HIV in Ethiopia. Int J Ment Health Syst. 2016;10:29.

55. Tran BX. Quality of life outcomes of antiretroviral treatment for HIV/AIDS patients in Vietnam. PLoS ONE. 2012;7(7):e41062.

56. Ahmed A, Saqlain M, Akhtar N, Hashmi FK, Blebil A, Dujaili J, Umair MM, Bukhsh A. Psychometric assessment of translated Urdu version of WHOQOL-HIV BREF among patients living with HIV. 2020.

57. Hsiung PC, Fang $\mathrm{CT}$, Wu CH, et al. Validation of the WHOQOL-HIV BREF among HIV-infected patients in Taiwan. AIDS Care. 2011;23(8):1035-42.

58. Zhu Y, Liu J, Qu B. Psychometric properties of the Chinese version of the WHOQOL-HIV BREF to assess quality of life among people living with HIV/ AIDS: a cross-sectional study. BMJ Open. 2017;7(8):e016382.
59. Cai NQ Xu DL Hao YT Revision of Chinese version of WHOQOL-HIV BREF and assessment of psychometric properties. Annual meeting of Chinese health statistics. 2011

60. Chen XL, Jia WD, Cen YW, et al. Reliability and validity of WHOQOL-HIV BREF for AIDS patients. China AIDS STD. 2010;3:239-42.

61. Luo X, Lei XB, Tian L, Liu LJ, Pan DM, Lian DY. Evaluation of reliability and validity of Chinese version of World Health Organization Quality of life for HIV/AIDS patients. Infect Dis Inform. 2014;5:289-91.

62. Liu WM, Wang J, Liu BY, et al. Technical report on the localization of WHOQOL-HIV. Clinical Epidemiology Branch of Chinese Medical Association. In: Proceedings of the 10th annual meeting of clinical epidemiology of Chinese Medical Association and the 2nd World Federation of Chinese Medicine Societies clinical efficacy evaluation Symposium. 2008. p. $125-128$.

63. Akinboro AO, Akinyemi SO, Olaitan PB, et al. Quality of life of Nigerians living with human immunodeficiency virus. Pan Afr Med J. 2014;18:234.

64. Thompson HM, Reisner SL, VanKim N, Raymond HF. Quality-of-life measurement: assessing the WHOQOL-BREF scale in a sample of HighHIV-risk transgender women in San Francisco, California. Int J Transgend. 2015;16(1):36-48.

65. Smith MY, Feldman J, Kelly P, DeHovitz JA, Chirgwin K, Minkoff H. Healthrelated quality of life of HIV-infected women: evidence for the reliability, validity and responsiveness of the medical outcomes study short-form 20. Qual Life Res. 1996;5(1):47-55.

66. Remple VP, Hilton BA, Ratner PA, Burdge DR. Psychometric assessment of the multidimensional quality of life questionnaire for persons with HIV/AIDS (MQOL-HIV) in a sample of HIV-infected women. Qual Life Res. 2004;13(5):947-57.

67. Smith KW, Avis NE, Mayer KH, Swislow L. Use of the MQoL-HIV with asymptomatic HIV-positive patients. Qual Life Res. 1997;6(6):555-60

68. Watanabe M, Nishimura K, Inoue T, Kimura S, Oka S. QoL Research Group of the AIDS Clinical Centre and Eight Regional AIDS Treatment Hospitals in Japan. A discriminative study of health-related quality of life assessment in HIV-1-infected persons living in Japan using the Multidimensional Quality of Life Questionnaire for persons with HIV/AIDS. Int J STD AIDS. 2004;15(2):107-15.

69. Bucciardini R, Murri R, Guarinieri M, et al. ISSQoL: a new questionnaire for evaluating the quality of life of people living with HIV in the HAART era. Qual Life Res. 2006;15(3):377-90.

70. Brown G, Mikołajczak G, Lyons A, et al. Development and validation of PozQoL: a scale to assess quality of life of PLHIV. BMC Public Health. 2018;18(1):527.

71. Herrmann S, McKinnon E, Hyland NB, et al. HIV-related stigma and physical symptoms have a persistent influence on health-related quality of life in Australians with HIV infection. Health Qual Life Outcomes. 2013;11:56.

72. Riley ED, Bangsberg DR, Perry S, Clark RA, Moss AR, Wu AW. Reliability and validity of the SF-36 in HIV-infected homeless and marginally housed individuals. Qual Life Res. 2003;12(8):1051-8.

73. Turner-Bowker DM, Saris-Baglama RN, DeRosa MA, Giovannetti ER, Jensen RE, Wu AW. A computerized adaptive version of the SF-36 is feasible for clinic and Internet administration in adults with HIV. AIDS Care. 2012;24(7):886-96.

74. Zhang S. Study on quality of life and influencing factors of HIV/AIDS patients. Changsha: Central South University; 2011.

75. Pereira M, Canavarro MC. Reliability and validity of the European Portuguese version of the quality of life index EUROHIS-QOL-8 in HIV-infected patients. Rev Port Saúde Pública. 2015;33(2):183-7.

76. Holmes WC, Shea JA. Two approaches to measuring quality of life in the HIV/AIDS population: HAT-QoL and MOS-HIV. Qual Life Res. 1999:8(6):515-27.

77. Xiang FM, Li G, Chen XJ, et al. Establishment and evaluation of AIDS quality of life scale. In: Symposium on prevention and treatment of AIDS and 2006 annual meeting of Chinese society of traditional Chinese medicine. 2006.

78. Xiang FM. A research on questionnaire of quality of life with patients living with HIV/AIDS in China. Guangzhou: Guangzhou University of Chinese Medicine. 2005

79. Xiang FM, Li G, Chen XJ, et al. Comparative study on psychological characteristics of self-made quality of life scale and MOS-HIV scale. In: Symposium on prevention and treatment of AIDS and 2006 annual meeting of Chinese society of traditional Chinese medicine. Guangzhou. 2006. 
80. Meng YJ, Li NX, Chen JH, Song YC, Qian ZH. Development of a quality of life measure specific for Chinese people living with HIV/AIDS. China Epidemiol. 2007;28(11):1081-4.

81. Su FJ. Development of quality of life scale for HIV/AIDS patients. Henan: Henan College of Traditional Chinese Medicine. 2007.

82. Guo XX, Zhang ML, Xu LR, et al. Preliminary development of HIV/AIDS quality of life scale. In: International symposium on prevention and treatment of AIDS. Chinese society of traditional Chinese medicine. 2007.

83. Davis EA, Pathak DS. Psychometric evaluation of four HIV disease-specific quality-of-life instruments. Ann Pharmacother. 2001;35(5):546-52.

84. Carabin H, Sonleitner NK, Keesee M, Shinault K. Quality of life measurement tools for people living with HIV/AIDS. J HIV/AIDS Soc Serv. 2008;7(1):71-83.

85. Skevington SM, Carse MS, Williams A. Validation of the WHOQOL-100: pain management improves quality of life for chronic pain patients. Clin J Pain. 2001;17(3):264-75.

86. Abbasi-Ghahramanloo A, Soltani-Kermanshahi M, Mansori K, et al. Comparison of SF-36 and WHOQoL-BREF in measuring quality of life in patients with type 2 diabetes. Int J Gen Med. 2020;13:497-506.

87. O'Brien N, Chi YL, Krause KR. Measuring Health outcomes in HIV: time to bring in the patient experience. Ann Glob Health. 2021;87(1):2.

88. von Steinbuechel N, Covic A, Polinder S, et al. Assessment of healthrelated quality of life after TBI: comparison of a disease-specific (QOLIBRI) with a generic (SF-36) instrument. Behav Neurol. 2016;2016:7928014.

89. Pequeno NP, de Araújo Cabral NL, Marchioni DM, Lima SC, de Oliveira LC Quality of life assessment instruments for adults: a systematic review of population-based studies. Health Qual Life Outcomes. 2020;18(1):1-3.

\section{Publisher's Note}

Springer Nature remains neutral with regard to jurisdictional claims in published maps and institutional affiliations.

- fast, convenient online submission

- thorough peer review by experienced researchers in your field

- rapid publication on acceptance

- support for research data, including large and complex data types

- gold Open Access which fosters wider collaboration and increased citations

- maximum visibility for your research: over $100 \mathrm{M}$ website views per year

At BMC, research is always in progress.

Learn more biomedcentral.com/submissions 